\title{
Self-Assembly of Anionic Polyoxometalate-Organic Architectures Based on Lacunary Phosphomolybdates and Pyridyl Ligands
}

\author{
Chifeng Li,${ }^{\dagger}$ Noritaka Mizuno, ${ }^{\dagger}$ Kazuya Yamaguchi, ${ }^{*}{ }^{\dagger}$ and Kosuke Suzuki ${ }^{*}, \dagger, \ddagger$
}

'Department of Applied Chemistry, School of Engineering, The University of Tokyo, 7-3-1 Hongo, Bunkyo-ku, Tokyo 113-8656, Japan.

*Precursory Research for Embryonic Science and Technology (PRESTO), Japan Science and Technology Agency (JST), 4-1-8 Honcho, Kawaguchi, Saitama 332-0012, Japan.

Instruments. CSI mass spectra were recorded on a JEOL JMS-T100CS instrument. ESI mass spectra were recorded on a Waters Xevo G2-XS QTof instrument. FT-IR spectra were measured on JASCO FT/IR-4100 instrument using KCl discs. NMR spectra were recorded on a JEOL ECA-500 spectrometer $\left({ }^{1} \mathrm{H}, 500.16 \mathrm{MHz} ;{ }^{31} \mathrm{P}, 202.47 \mathrm{MHz}\right)$ using $5 \mathrm{~mm}$ tubes. Chemical shifts $(\delta)$ are reported in ppm downfield from TMS for ${ }^{1} \mathrm{H}$ NMR spectra and upfield from $\mathrm{H}_{3} \mathrm{PO}_{4}$ (solvent, $\mathrm{D}_{2} \mathrm{O}$ ) for ${ }^{31} \mathrm{P}$ NMR spectra, respectively. UV-Vis spectra were recorded on a JASCO V-570 instrument using quartz cells with a 1.0 $\mathrm{cm}$ optical path length. TG-DTA was performed on a Rigaku Thermo plus TG 8120 instrument. ICP-AES analyses for P and Mo were performed with Shimadzu ICPS-8100 instrument. Elemental analyses for $\mathrm{C}, \mathrm{H}$ and $\mathrm{N}$ were performed on Elementar vario MICRO cube at the Elemental Analysis Centre of the School of Science of the University of Tokyo.

Materials. Sodium molybdate dihydrate (Wako), sodium dihydrogen phosphate dihydrate (Wako), hydrochloric acid (Kanto Chemical), tetra- $n$-butyl ammonium bromide (TBABr, TCI), tetraphenyl phosphonium bromide (TPPBr, TCI), N,N-dimethylacetamide (DMA, Kanto Chemical), acetone (Kanto Chemical), acetonitrile (Kanto Chemical), nitromethane (TCI), 1,2-dichloroethane (Kanto Chemical), 1,4-dioxane (Kanto Chemical), ethyl acetate (Kanto Chemical), diethyl ether (Kanto Chemical), pyridine (py, Kanto Chemical), 4,4'-bipyridine (bpy, TCI), 5,10,15,20-tetra(4-pyridyl)porphyrin (tpyp, TCI), acenaphthenequinone (TCI), 1,4-dihydroxynaphthalene (TCI), 1,4-naphthoquinone (TCI), 1,8-naphthalic anhydride (TCI) and deuterated solvents (dimethyl sulfoxide- $d_{6}$, acetonitrile- $d_{3}$, nitromethane- $d_{3}$ and pyridine- $d_{5}$ ) (Kanto Chemical) were used as received.

X-ray crystallography. Single-crystal X-ray diffraction measurements were made on a Rigaku MicroMax-007 Saturn 724 CCD detector with graphic monochromated Mo K $\alpha$ radiation $(\lambda=0.71069 \AA$, $50 \mathrm{kV}, 24 \mathrm{~mA}$ ) at $123 \mathrm{~K}$. The data were collected and processed using CrystalClear ${ }^{\mathrm{S} 1, \mathrm{~S} 2}$ and HKL2000. ${ }^{\mathrm{S} 3}$ Neutral scattering factors were obtained from the standard source. In the reduction of data, Lorentz and polarization corrections were made. The structural analyses were performed using CrystalStructure ${ }^{\mathrm{S} 4}$ and WinGX. $^{\mathrm{S} 5}$ All structures were solved by SHELXS-97 (direct methods) and refined by SHELXL-2014. ${ }^{\mathrm{S6}, \mathrm{S} 7}$ The metal atoms (P, Mo) and oxygen atoms in the POM frameworks, ligands and TPP cations were refined anisotropically. Solvents of crystallization and TBA cations were refined 
isotropically. Large $R_{1}$ and $w R_{2}$ values for TPP-III•ANQ were likely due to the severe disorder of TPP cations and solvent molecules. The highly disordered TBA cations and solvent molecules in TBA-I, TBA-II and TPP-III•ANQ were omitted by use of SQUEEZE program. ${ }^{\text {S8 }}$

Bond valence sum (BVS) calculations. The BVS values were calculated by the expression for the variation of the length $r_{i j}$ of a bond between two atoms $i$ and $j$ in observed crystal with valence $V_{i}$ using equation:

$$
V_{i}=\sum_{j} \exp \left(\frac{r_{0}^{\prime}-r_{i j}}{B}\right)
$$

where $\mathrm{B}$ is a constant equal to $0.37 \AA, r_{0}^{\prime}$ is bond valence parameter for a given atom pair. ${ }^{\mathrm{S}, \mathrm{S} 10}$

Synthesis of TBA-I. $\mathrm{Na}_{2} \mathrm{MoO}_{4} \cdot 2 \mathrm{H}_{2} \mathrm{O}(8.72 \mathrm{~g}, 36.0 \mathrm{mmol})$ was dissolved in water $\left(200 \mathrm{~mL}, 5^{\circ} \mathrm{C}\right)$, and $\mathrm{NaH}_{2} \mathrm{PO}_{4} \cdot 2 \mathrm{H}_{2} \mathrm{O}(624 \mathrm{mg}, 4.00 \mathrm{mmol})$ was added. After the $\mathrm{pH}$ of the solution was adjusted to 1.0 by adding $37 \% \mathrm{HCl}$, TBABr $(12.9 \mathrm{~g}, 40.0 \mathrm{mmol})$ was added, and the solution was vigorously stirred at $5{ }^{\circ} \mathrm{C}$ for $1 \mathrm{~min}$. The precipitate formed was filtered off and washed with cold water to afford a pale-yellow powder of $\mathrm{TBA}_{3}\left[\mathrm{H}_{6} \mathrm{PMo}_{9} \mathrm{O}_{34}\right]\left(9.70 \mathrm{~g}, 86 \%\right.$ purity, $96 \%$ yield based on $\left.\mathrm{NaH}_{2} \mathrm{PO}_{4} \cdot 2 \mathrm{H}_{2} \mathrm{O}\right)$. After the addition of pyridine $(5.0 \mathrm{~mL})$ to crude $\mathrm{TBA}_{3}\left[\mathrm{H}_{6} \mathrm{PMo}_{9} \mathrm{O}_{34}\right](1.00 \mathrm{~g})$, the mixture was stirred vigorously for $30 \mathrm{~min}$, and then it was filtered with a membrane filter. The residue was washed with pyridine $(1.0 \mathrm{~mL})$, then it was dissolved in a mixture of acetonitrile/pyridine $(9 / 1, v / v, 5.00 \mathrm{~mL})$. Excess ethyl acetate was added to the solution and the precipitate formed was filtered with a membrane filter, washed with ethyl acetate and dried in vacuo affording TBA-I as a white powder $(515 \mathrm{mg}, 53.3 \%$ yield based on $\left.\mathrm{TBA}_{3}\left[\mathrm{H}_{6} \mathrm{PMo}_{9} \mathrm{O}_{34}\right]\right)$. Colourless single crystals suitable for $\mathrm{X}$-ray crystallographic analysis were obtained in two days by vapour diffusion of ethyl acetate into a solution of TBA-I in a mixture of DMA/pyridine $(9 / 1, v / v)$ at $25{ }^{\circ} \mathrm{C}$. Positive-ion MS (ESI, acetonitrile): $\mathrm{m} / \mathrm{z} 2360.075$ (calcd. 2360.110 for $\left[\mathrm{TBA}_{4} \mathrm{PMo}_{9} \mathrm{O}_{31}\right]^{+}$). Positive-ion MS (CSI, acetonitrile/pyridine, v/v, 9/1): $\mathrm{m} / z 2360.2$ (calcd. 2360.1 for [TBA $\left.{ }_{4} \mathrm{PMo}_{9} \mathrm{O}_{31}\right]^{+}$), $\mathrm{m} / \mathrm{z} 2401.2$ (calcd. 2401.1 for $\left[\mathrm{TBA}_{4} \mathrm{PMo}_{9} \mathrm{O}_{31}\left(\mathrm{CH}_{3} \mathrm{CN}\right.\right.$ )] ${ }^{+}$), 2439.3 (calcd. 2439.2 for $\left.\left[\mathrm{TBA}_{4} \mathrm{PMo}_{9} \mathrm{O}_{31}\left(\mathrm{C}_{5} \mathrm{H}_{5} \mathrm{~N}\right)\right]^{+}\right) .{ }^{31} \mathrm{P}$ NMR $\left(202.47 \mathrm{MHz}\right.$, acetonitrile- $\left.d_{3}\right): \delta=-0.26 \mathrm{ppm} .{ }^{1} \mathrm{H}$ NMR (500.16 MHz, acetonitrile- $\left.d_{3}\right): 8.81(\mathrm{~d}, J=5.15 \mathrm{~Hz}, 6 \mathrm{H}), 7.83(\mathrm{tt}, J=7.73,1.72 \mathrm{~Hz}, 3 \mathrm{H}), 7.36$ (t, $J=7.45 \mathrm{~Hz}$, $6 \mathrm{H}), 3.11(\mathrm{~m}, 24 \mathrm{H}), 2.93(\mathrm{~s}, 3 \mathrm{H}), 2.81(\mathrm{~s}, 3 \mathrm{H}), 1.96(\mathrm{~s}, 3 \mathrm{H}), 1.60(\mathrm{~m}, 24 \mathrm{H}), 1.35(\mathrm{~m}, 24 \mathrm{H}), 0.95(\mathrm{~m}$, 36H). IR ( $\mathrm{KCl}$ pellet, $\mathrm{cm}^{-1}$ ): 1633, 1605, 11483, 1446, 1380, 1219, 1152, 1055, 1010, 933, 919, 898, $872,846,763,721,668,650,633$, 594, 570, 519. Elemental analysis calcd (\%) for $\mathrm{TBA}_{3}\left[\mathrm{~A}-\alpha-\mathrm{PMo}_{9} \mathrm{O}_{31}(\mathbf{p y})_{3}\right] \cdot \mathrm{DMA}\left(\mathrm{C}_{67} \mathrm{H}_{132} \mathrm{Mo}_{9} \mathrm{~N}_{7} \mathrm{O}_{32} \mathrm{P}\right): \mathrm{C}, 32.95 ; \mathrm{H}, 5.45 ; \mathrm{N}, 4.01 ; \mathrm{Mo}, 35.36 ; \mathrm{P}, 1.27$; found: $\mathrm{C}, 32.69 ; \mathrm{H}, 5.61 ; \mathrm{N}, 4.00 ; \mathrm{Mo}, 36.34 ; \mathrm{P}, 1.28$.

Synthesis of TPP-I. TPP $3\left[\mathrm{H}_{6} \mathrm{PMo}_{9} \mathrm{O}_{34}\right]$ was synthesised using a similar method to that used for $\mathrm{TBA}_{3}\left[\mathrm{H}_{6} \mathrm{PMo}_{9} \mathrm{O}_{34}\right]$ but using TPPBr instead of TBABr (TPPBr was added as a solution in a mixture of acetonitrile $\left./ \mathrm{H}_{2} \mathrm{O}\right)$. After addition of pyridine $(5.0 \mathrm{~mL})$ to $\mathrm{TPP}_{3}\left[\mathrm{H}_{6} \mathrm{PMo}_{9} \mathrm{O}_{34}\right](1.00 \mathrm{~g})$, the mixture was stirred vigorously for $1 \mathrm{~min}$ and, then, filtered through a membrane filter. Acetone $(30.0 \mathrm{~mL})$ was added to the filtrate, and the precipitate formed was filtered with a membrane filter, washed with acetone (5.0 $\mathrm{mL}$ ) and dried in vacuo, affording TPP-I as a white powder (770 $\mathrm{mg}, 67.7 \%$ yield). Colourless single 
crystals suitable for X-ray crystallographic analysis were obtained in two days by vapour diffusion of acetone into a pyridine solution of TPP-I at $25{ }^{\circ} \mathrm{C}$. Positive-ion MS (ESI, acetonitrile): $\mathrm{m} / \mathrm{z} 2748.431$ (calcd. 2748.493 for $\left.\left[\mathrm{TPP}_{4} \mathrm{PMo}_{9} \mathrm{O}_{31}\right]^{+}\right) .{ }^{31} \mathrm{P}$ NMR $\left(202.47 \mathrm{MHz}\right.$, acetonitrile- $\left.d_{3}\right): \delta=23.62$ (TPP cation) and $-0.25 \mathrm{ppm} .{ }^{1} \mathrm{H}$ NMR (500.16 MHz, acetonitrile- $\left.d_{3}\right): 8.69(\mathrm{br}, 6 \mathrm{H}), 7.90(\mathrm{~m}, 12 \mathrm{H}), 7.77(\mathrm{~m}, 3 \mathrm{H})$, 7.75-7.70 (m, 24H), 7.60-7.63 (m, 24H), 7.34-7.29 (m, 6H). IR (KCl pellet, $\mathrm{cm}^{-1}$ ): 3418, 3061, 2924, 2360, 1705, 1634, 1604, 1586, 1484, 1437, 1356, 1339, 1318, 1217, 1189, 1165, 1148, 1109, 1054, 1007, $931,916,898,873,842,768,722,689,650,633,616,595,569,527,495,464,430,370$. Elemental analysis calcd (\%) for $\mathrm{TPP}_{3}\left[\mathrm{~A}-\alpha-\mathrm{PMo}_{9} \mathrm{O}_{31}(\mathbf{p y})_{3}\right] \cdot 2 \mathbf{p y} \cdot 1 \mathrm{H}_{2} \mathrm{O}\left(\mathrm{C}_{97} \mathrm{H}_{87} \mathrm{Mo}_{9} \mathrm{~N}_{5} \mathrm{O}_{32} \mathrm{P}_{4}\right)$ : C, 41.28; H, 3.11; N, 2.48; Mo, 30.60; P, 4.39; found: C, 41.45; H, 3.52; N, 2.52; Mo, 31.10; P, 4.45.

Synthesis of TBA-II. To an acetonitrile $(10.0 \mathrm{~mL})$ solution of 4,4'-bipyridine (23.4 $\mathrm{mg}, 150 \mu \mathrm{mol})$, TBA-I (244 mg, $100 \mu \mathrm{mol})$ was added. After stirring for $1 \mathrm{~h}, 1,4$-dioxane $(20.0 \mathrm{~mL})$ was added to the solution. The precipitate formed was filtered off and the filtrate was kept in the opened vessel at room temperature for 2 days affording TBA-II as colourless crystals (200 mg, 79.6\% yield). Colourless single crystals suitable for X-ray crystallographic analysis were obtained in 2 days by vapour diffusion of 1,4-dioxane into a nitromethane solution of TBA-II at $25{ }^{\circ} \mathrm{C}$. Positive-ion MS (ESI, acetonitrile): $\mathrm{m} / \mathrm{z}$ 1810.569 (calcd. 1810.571 for $\left[\mathrm{TBA}_{9}\left(\mathrm{PMo}_{9} \mathrm{O}_{31}\right)_{2}(\mathbf{b p y})_{3}\right]^{3+} ; \mathrm{m} / \mathrm{z} 2594.832$ (calcd. 2594.713 for $\left.\left[\mathrm{TBA}_{8}\left(\mathrm{PMo}_{9} \mathrm{O}_{31}\right)_{2}(\mathbf{b p y})_{3}\right]^{2+}\right) .{ }^{31} \mathrm{P}$ NMR $\left(202.47 \mathrm{MHz}\right.$, nitromethane- $\left.d_{3}\right): \delta=-0.70 \mathrm{ppm} .{ }^{1} \mathrm{H}$ NMR (500.16 MHz, nitromethane- $\left.d_{3}\right)$ : $9.14(\mathrm{br}, 12 \mathrm{H}), 7.67(\mathrm{br}, 12 \mathrm{H}), 3.57(\mathrm{~s}, 24 \mathrm{H}), 3.25(\mathrm{~m}, 48 \mathrm{H}), 1.70(\mathrm{~m}, 48 \mathrm{H}), 1.38$ (m, 48H), 0.94 (m, 72H). IR (KCl pellet, $\left.\mathrm{cm}^{-1}\right): 3418,2959,2925,2871,2852,2360,2342,1700,1653$, $1610,1534,1507,1483,1469,1410,1379,1315,1286,1254,1220,1151,1120,1056,1012,953,933$, $922,901,870,842,809,763,721,636,594,569,519,494,430,369,339$. Elemental analysis calcd (\%) for $\mathrm{TBA}_{6}\left[\left(\mathrm{~A}-\alpha-\mathrm{PMo}_{9} \mathrm{O}_{31}\right)_{2}(\mathbf{b p y})_{3}\right] \cdot 3(1,4-$ dioxane $) \cdot 3 \mathrm{H}_{2} \mathrm{O}\left(\mathrm{C}_{138} \mathrm{H}_{270} \mathrm{~N}_{12} \mathrm{O}_{71} \mathrm{P}_{2} \mathrm{Mo}_{18}\right): \mathrm{C}, 33.00 ; \mathrm{H}, 5.42 ; \mathrm{N}$, 3.35; Mo, 34.38; P, 1.23; found: C, 33.05; H, 5.37; N, 3.29; Mo, 35.45; P, 1.25.

Synthesis of TPP-III. To a DMA/DCE (1/1,v/v; $6 \mathrm{~mL}$ ) solution of 5,10,15,20-tetra(4-pyridyl)porphyrin (12.4 mg, $20.0 \mu \mathrm{mol})$, TPP-I (112 mg, $40.0 \mu \mathrm{mol})$ was added. After stirring at $50{ }^{\circ} \mathrm{C}$ for $1 \mathrm{~h}$, the solution was cooled to room temperature and, upon addition of toluene and standing at $25{ }^{\circ} \mathrm{C}$, purple crystals of TPP-III were obtained in 2 days (95.6 mg, 80.7\% yield). Single crystals suitable for X-ray crystallography were successfully obtained by recrystallisation of TPP-III from a mixture of DMA/DCE $(1 / 1, v / v)$ and diethyl ether in the presence of pyridine (100 equiv with respect to TPP-III) at $25^{\circ} \mathrm{C}$ in 2 days. Positive-ion MS (ESI, acetonitrile): $\mathrm{m} / \mathrm{z}$ 3057.365, (calcd. 3057.358 for $\left.\left[\mathrm{TPP}_{16}\left(\mathrm{PMo}_{9} \mathrm{O}_{31}\right)_{4}(\mathbf{t p y p})_{2}\right]^{4+}\right)$. IR $\left(\mathrm{KCl}\right.$ pellet, $\left.\mathrm{cm}^{-1}\right): 3418,3079,3057,2360,2342,1630,1610,1586$, 1559, 1483, 1436, 1415, 1354, 1339, 1318, 1217, 1189, 1164, 1108, 1054, 1010, 971, 954, 932, 918, 899, $872,842,802,766,721,688,615,593,570,527,428,369,360$. Elemental analysis calcd (\%) for $\mathrm{TPP}_{12}\left[\left(\mathrm{~A}-\alpha-\mathrm{PMo}_{9} \mathrm{O}_{31}\right)_{4}(\mathbf{t p y p})_{2}(\mathbf{p y})_{4}\right] \cdot 5 \mathrm{DMA} \cdot 12 \mathrm{H}_{2} \mathrm{O}\left(\mathrm{C}_{408} \mathrm{H}_{381} \mathrm{Mo}_{36} \mathrm{~N}_{25} \mathrm{O}_{141} \mathrm{P}_{16}\right): \mathrm{C}, 41.39 ; \mathrm{H}, 3.24 ; \mathrm{N}$, 2.96; Mo, 29.18; P, 4.19; found: C, 41.17; H, 3.24; N, 2.98; Mo, 28.95; P, 4.14.

Synthesis of TBA-III. To a DMA/DCE (1/1, v/v; $4 \mathrm{~mL})$ solution of 5,10,15,20-tetra(4-pyridyl)porphyrin $(24.8 \mathrm{mg}, 40.0 \mu \mathrm{mol})$, TBA-I $(195 \mathrm{mg}, 80.0 \mu \mathrm{mol})$ was added. After stirring at $50{ }^{\circ} \mathrm{C}$ for $1 \mathrm{~h}$, the solution 
was cooled to room temperature, and following addition of toluene, purple crystals of TBA-III were obtained at $25{ }^{\circ} \mathrm{C}$ in 2 days (130 mg, 61.4\% yield). Single crystals suitable for X-ray crystallography were successfully obtained by recrystallisation of TBA-III from a mixture of DMA/DCE $(1 / 1, v / v)$ and ethyl acetate in the presence of pyridine (100 equiv with respect to TBA-III) at $25{ }^{\circ} \mathrm{C}$ in 2 days. Positive-ion MS (ESI, acetonitrile): $m / z$ 2669.722, (calcd. 2669.725 for $\left.\left[\mathrm{TBA}_{16}\left(\mathrm{PMo}_{9} \mathrm{O}_{31}\right)_{4}(\text { tpyp })_{2}\right]^{4+}\right) .{ }^{31} \mathrm{P}$ $\operatorname{NMR}\left(202.47 \mathrm{MHz}\right.$, acetonitrile- $\left.d_{3}\right): \delta=-0.24 \mathrm{ppm} .{ }^{1} \mathrm{H}$ NMR (500.16 MHz, acetonitrile- $\left.d_{3}\right): 9.67$ (d, $J=$ $5.15 \mathrm{~Hz}, 4 \mathrm{H}), 9.41(\mathrm{~d}, J=5.44 \mathrm{~Hz}, 4 \mathrm{H}), 9.22$ (d, $J=5.15 \mathrm{~Hz}, 4 \mathrm{H}), 9.14$ (d, $J=4.58 \mathrm{~Hz}, 8 \mathrm{H}), 9.11(\mathrm{~d}, J=$ $5.44 \mathrm{~Hz}, 4 \mathrm{H}), 9.06(\mathrm{~d}, J=5.44 \mathrm{~Hz}, 4 \mathrm{H}), 8.63-8.58(\mathrm{~m}, 10 \mathrm{H}), 8.54(\mathrm{~d}, J=5.15 \mathrm{~Hz}, 4 \mathrm{H}), 8.51(\mathrm{~d}, J=4.58$ $\mathrm{Hz}, 4 \mathrm{H}), 8.30(\mathrm{t}, J=6.87 \mathrm{~Hz}, 4 \mathrm{H}), 8.18(\mathrm{t}, J=4.87 \mathrm{~Hz}, 8 \mathrm{H}), 7.76-7.72(\mathrm{~m}, 12 \mathrm{H}), 7.36(\mathrm{t}, J=7.02 \mathrm{~Hz}$, 4H), 6.39 (d, $J=4.01 \mathrm{~Hz}, 4 \mathrm{H}), 5.30(\mathrm{t}, J=5.44 \mathrm{~Hz}, 4 \mathrm{H}), 4.40(\mathrm{t}, J=5.44 \mathrm{~Hz}, 4 \mathrm{H}), 3.12(\mathrm{~m}, 96 \mathrm{H}), 1.61$ (m, 96H), 1.35 (m, 96H), 0.92 (m, 144H). IR ( $\mathrm{KCl}$ pellet, $\left.\mathrm{cm}^{-1}\right):$ 3409, 3089, 3049, 2959, 2931, 2871, 2360, 2341, 1633, 1611, 1558, 1484, 1446, 1415, 1400, 1380, 1352, 1261, 1217, 1190, 1153, 1055, 1011, 933, 920, 899, 872, 846, 802, 762, 722, 680, 593, 569, 520, 494, 369. UV-Vis (acetonitrile): $\lambda_{\max }(\varepsilon$, $\left.\mathrm{M}^{-1} \cdot \mathrm{cm}^{-1}\right)=414 \mathrm{~nm}\left(6.09 \times 10^{5}\right), 518 \mathrm{~nm}\left(3.66 \times 10^{4}\right), 552 \mathrm{~nm}\left(1.64 \times 10^{4}\right), 590 \mathrm{~nm}\left(1.37 \times 10^{4}\right), 646$ $\mathrm{nm} \quad\left(6.92 \times 10^{3}\right)$. Elemental analysis calcd $(\%)$ for $\mathrm{TBA}_{10} \mathrm{H}_{2}\left[\left(\mathrm{~A}-\alpha-\mathrm{PMo}_{9} \mathrm{O}_{31}\right)_{4}(\text { tpyp })_{2}(\mathbf{p y})_{4}\right] \cdot 2$ py $7 \mathrm{DMA} \cdot 5 \mathrm{DCE}\left(\mathrm{C}_{308} \mathrm{H}_{513} \mathrm{~N}_{39} \mathrm{Cl}_{10} \mathrm{P}_{4} \mathrm{Mo}_{36} \mathrm{O}_{131}\right): \mathrm{C}, 34.28 ; \mathrm{H}$, 4.79; N, 5.06; Mo, 32.00; P, 1.15; found: C, 34.69; H, 4.97; N, 5.04; Mo, 32.25; P, 1.15 .

Synthesis of TPP-III•ANQ. To a DMA/DCE (1/1, $v / v ; 6 \mathrm{~mL})$ solution of TPP-III (118.4 mg, $10 \mu \mathrm{mol})$, acenaphthenequinone $(18.2 \mathrm{mg}, 100 \mu \mathrm{mol})$ and pyridine $(79.1 \mathrm{mg}, 1 \mathrm{mmol})$ were added. After stirring at room temperature for $1 \mathrm{~h}$, ethyl acetate was added to the solution. Brown crystals of TPP-III•ANQ suitable for X-ray crystallography were obtained at $25{ }^{\circ} \mathrm{C}$ after 3 days $(86.0 \mathrm{mg}, 70.1 \%$ yield). Positive-ion MS (ESI, acetonitrile): $\mathrm{m} / \mathrm{z} \quad 3102.103$, (calcd. 3102.109 for $\left.\left[\mathrm{TPP}_{16}\left(\mathrm{PMo}_{9} \mathrm{O}_{31}\right)_{4}(\mathbf{t p y p})_{2}(\mathbf{A N Q})\right]^{4+}\right)$. IR $\left(\mathrm{KCl}\right.$ pellet, $\left.\mathrm{cm}^{-1}\right): 3418,3087,3058,2361,2342,1725,1631$, 1610, 1483, 1437, 1417, 1371, 1353, 1318, 1242, 1218, 1189, 1164, 1108, 1053, 1011, 971, 932, 919, $899,872,843,804,765,721,688,595,571,527,494,429,403,370,333$. Elemental analysis calcd (\%) for $\quad \operatorname{TPP}_{12}\left[\left(\mathrm{~A}-\alpha-\mathrm{PMo}_{9} \mathrm{O}_{31}\right)_{4}(\text { tpyp })_{2}(\mathbf{p y})_{4}(\mathbf{A N Q})\right] \cdot 4 \mathrm{DMA} \cdot 3($ ethyl $\quad$ acetate $) \cdot 15 \mathrm{H}_{2} \mathrm{O}$ $\left(\mathrm{C}_{428} \mathrm{H}_{408} \mathrm{~N}_{24} \mathrm{P}_{16} \mathrm{Mo}_{36} \mathrm{O}_{151}\right)$ : C, 41.95; H, 3.36; N, 2.74; Mo, 28.19; P, 4.04; found: C, 41.79; H, 3.46; N, 2.72; Mo, 28.88; P, 4.01 . 
Table S1. Crystal data for TBA-I, TPP-I and TBA-II.

\begin{tabular}{llll}
\hline & TBA-I & TPP-I & TBA-II \\
\hline Formula & $\mathrm{C}_{63} \mathrm{H}_{15} \mathrm{Mog}_{9} \mathrm{~N}_{6} \mathrm{O}_{31} \mathrm{P}$ & $\mathrm{C}_{261} \mathrm{H}_{225} \mathrm{Mo}_{27} \mathrm{~N}_{9} \mathrm{O}_{93} \mathrm{P}_{12}$ & $\mathrm{C}_{94} \mathrm{H}_{24} \mathrm{Mo}_{18} \mathrm{~N}_{10} \mathrm{O}_{62} \mathrm{P}_{2}$ \\
Fw $\left(\mathrm{g} \mathrm{mol}{ }^{-1}\right)$ & 2246.24 & 7937.51 & 4074.09 \\
Crystal system & orthorhombic & trigonal & orthorhombic \\
Space group & Pna2 $_{1}($ No. 33) & $R-3 c($ No. 167) & Pccn (No. 56) \\
$a(\AA)$ & $18.4306(2)$ & $25.0750(2)$ & $34.5995(3)$ \\
$b(\AA)$ & $21.7192(2)$ & $25.0750(2)$ & $21.4956(2)$ \\
$c(\AA)$ & $26.7317(3)$ & $57.2801(7)$ & $27.1188(2)$ \\
$\alpha(\mathrm{deg})$ & 90 & 90 & 90 \\
$\beta(\mathrm{deg})$ & 90 & 90 & 90 \\
$\gamma(\mathrm{deg})$ & 90 & 120 & 90 \\
$V\left(\AA^{3}\right)$ & $10700.64(19)$ & $31190.1(6)$ & $20169.3(3)$ \\
$Z$ & 4 & 12 & 4 \\
$R_{1}{ }^{[\mathrm{a}]}[I>2 \sigma(I)]$ & 0.0538 & 0.0560 & 0.0816 \\
$w R_{2}{ }^{\text {la }}$ & 0.1689 & 0.1865 & 0.2702 \\
$\mathrm{GOF}$ & 1.068 & 1.075 & 1.030 \\
$\rho_{\text {calcd }}\left(\mathrm{g} \mathrm{cm}{ }^{-3}\right)$ & 1.394 & 1.690 & 1.342 \\
Temp $(\mathrm{K})$ & 123 & 123 & 123 \\
\hline
\end{tabular}

[a] $R_{1}=\Sigma|| F_{\mathrm{o}}|-| F_{\mathrm{c}}|| / \Sigma\left|F_{\mathrm{o}}\right|, w R_{2}=\left\{\Sigma\left[w\left(F_{\mathrm{o}}{ }^{2}-F_{\mathrm{c}}{ }^{2}\right)\right] / \Sigma\left[w\left(F_{\mathrm{o}}{ }^{2}\right)^{2}\right]\right\}^{1 / 2}$.

Table S2. Crystal data for TPP-III and TPP-III•ANQ.

\begin{tabular}{|c|c|c|}
\hline & TPP-III & TPP-III•ANQ \\
\hline Formula & $\mathrm{C}_{320} \mathrm{H}_{261} \mathrm{Mo}_{36} \mathrm{~N}_{21} \mathrm{O}_{125} \mathrm{P}_{13}$ & $\mathrm{C}_{280} \mathrm{H}_{218} \mathrm{Mo}_{36} \mathrm{~N}_{20} \mathrm{O}_{126} \mathrm{P}_{11}$ \\
\hline $\mathrm{FW}\left(\mathrm{g} \mathrm{mol}^{-1}\right)$ & 10256.93 & 9673.24 \\
\hline Crystal system & monoclinic & monoclinic \\
\hline Space group & $C 2$ (No. 5) & $P 2_{1} / c$ (No. 14$)$ \\
\hline$a(\AA)$ & $38.3334(2)$ & $30.0613(2)$ \\
\hline$b(\AA)$ & $33.8624(3)$ & $27.2811(2)$ \\
\hline$c(\AA)$ & $42.0755(3)$ & $67.4589(7)$ \\
\hline$\alpha(\mathrm{deg})$ & 90 & 90 \\
\hline$\beta(\operatorname{deg})$ & $101.2820(10)$ & $98.1644(3)$ \\
\hline$\gamma(\operatorname{deg})$ & 90 & 90 \\
\hline$V\left(\AA^{3}\right)$ & $53561.1(7)$ & $54762.7(8)$ \\
\hline$Z$ & 4 & 4 \\
\hline$R_{1}^{[\mathrm{a}]}[I>2 \sigma(I)]$ & 0.0622 & 0.1012 \\
\hline$w R_{2}^{\lfloor\mathrm{a}\rfloor}$ & 0.1951 & 0.3085 \\
\hline GOF & 1.011 & 1.036 \\
\hline$\rho_{\text {calcd }}\left(\mathrm{g} \mathrm{cm}^{-3}\right)$ & 1.272 & 1.173 \\
\hline Temp (K) & 123 & 123 \\
\hline
\end{tabular}

[a] $R_{1}=\Sigma|| F_{\mathrm{o}}|-| F_{\mathrm{c}}|| \Sigma\left|F_{\mathrm{o}}\right|, w R_{2}=\left\{\Sigma\left[w\left(F_{\mathrm{o}}{ }^{2}-F_{\mathrm{c}}{ }^{2}\right)\right] / \Sigma\left[w\left(F_{\mathrm{o}}{ }^{2}\right)^{2}\right]\right\}^{1 / 2}$. 
Table S3. BVS values for phosphorous, molybdenum and oxygen atoms of TBA-I.

\begin{tabular}{llllllll}
\hline P1 & 4.759 & O1 & 1.680 & O11 & 2.074 & O21 & 1.843 \\
Mo1 & 6.042 & O2 & 1.708 & O12 & 1.967 & O22 & 2.008 \\
Mo2 & 6.101 & O3 & 1.680 & O13 & 2.071 & O23 & 1.886 \\
Mo3 & 6.151 & O4 & 1.680 & O14 & 2.000 & O24 & 2.069 \\
Mo4 & 6.151 & O5 & 1.788 & O15 & 2.083 & O25 & 1.956 \\
Mo5 & 5.989 & O6 & 1.812 & O16 & 1.819 & O26 & 1.827 \\
Mo6 & 6.214 & O7 & 1.803 & O17 & 1.799 & O27 & 1.717 \\
Mo7 & 6.058 & O8 & 1.667 & O18 & 1.850 & O28 & 1.822 \\
Mo8 & 5.993 & O9 & 1.812 & O19 & 1.912 & O29 & 1.929 \\
Mo9 & 6.036 & O10 & 1.971 & O20 & 2.005 & O30 & 1.941 \\
& & & & & & O31 & 1.981 \\
\hline
\end{tabular}

Table S4. BVS values for phosphorous, molybdenum and oxygen atoms of TPP-I.

\begin{tabular}{llllllll}
\hline P1 & 4.718 & O1 & 1.867 & O5 & 1.828 & O9 & 1.917 \\
Mo1 & 6.088 & O2 & 2.077 & O6 & 2.005 & O10 & 1.745 \\
Mo2 & 6.103 & O3 & 1.750 & O7 & 1.783 & O11 & 1.973 \\
Mo3 & 6.077 & O4 & 1.717 & O8 & 2.039 & & \\
\hline
\end{tabular}

Table S5. BVS values for phosphorous, molybdenum and oxygen atoms of TBA-II.

\begin{tabular}{llllllll}
\hline P1 & 4.766 & O1 & 1.671 & O11 & 1.993 & O21 & 2.005 \\
Mo1 & 6.063 & O2 & 1.717 & O12 & 2.081 & O22 & 1.759 \\
Mo2 & 6.241 & O3 & 1.703 & O13 & 2.025 & O23 & 1.726 \\
Mo3 & 6.102 & O4 & 1.769 & O14 & 2.107 & O24 & 1.778 \\
Mo4 & 6.166 & O5 & 1.783 & O15 & 2.002 & O25 & 2.040 \\
Mo5 & 6.091 & O6 & 1.803 & O16 & 1.886 & O26 & 1.948 \\
Mo6 & 6.161 & O7 & 1.740 & O17 & 2.042 & O27 & 1.997 \\
Mo7 & 6.027 & O8 & 1.778 & O18 & 1.847 & O28 & 1.819 \\
Mo8 & 6.075 & O9 & 1.793 & O19 & 2.039 & O29 & 1.820 \\
Mo9 & 5.942 & O10 & 2.086 & O20 & 1.823 & O30 & 1.821 \\
& & & & & & O31 & 1.954 \\
\hline
\end{tabular}


Table S6. BVS values for phosphorous, molybdenum and oxygen of TPP-III.

\begin{tabular}{|c|c|c|c|c|c|c|c|}
\hline $\mathrm{P} 1$ & 4.703 & P2 & 4.741 & P3 & 4.591 & P4 & 4.794 \\
\hline Mo1 & 6.095 & Mo10 & 6.129 & Mo19 & 5.996 & Mo28 & 6.209 \\
\hline Mo2 & 6.214 & Mo11 & 5.783 & Mo20 & 5.850 & Mo29 & 5.772 \\
\hline Mo3 & 5.959 & Mo12 & 6.013 & Mo21 & 5.853 & Mo30 & 5.819 \\
\hline Mo4 & 5.984 & Mo13 & 5.854 & Mo22 & 6.162 & Mo31 & 5.844 \\
\hline Mo5 & 5.969 & Mo14 & 6.249 & Mo23 & 6.131 & Mo32 & 6.132 \\
\hline Mo6 & 6.020 & Mo15 & 5.942 & Mo24 & 5.761 & Mo33 & 6.021 \\
\hline Mo7 & 6.014 & Mo16 & 6.114 & Mo25 & 5.925 & Mo34 & 5.846 \\
\hline Mo8 & 5.920 & Mo17 & 6.085 & Mo26 & 5.858 & Mo35 & 5.816 \\
\hline Mo9 & 6.164 & Mo18 & 6.167 & Mo27 & 5.864 & Mo36 & 6.204 \\
\hline O1 & 1.750 & O32 & 1.847 & O63 & 1.545 & O94 & 1.827 \\
\hline $\mathrm{O} 2$ & 1.852 & O33 & 1.649 & O64 & 1.609 & O95 & 1.579 \\
\hline $\mathrm{O} 3$ & 1.788 & O34 & 1.698 & O65 & 1.537 & O96 & 1.635 \\
\hline O4 & 1.764 & O35 & 1.759 & O66 & 1.807 & O97 & 1.703 \\
\hline O5 & 1.635 & O36 & 1.847 & O67 & 1.740 & O98 & 1.812 \\
\hline O6 & 1.807 & O37 & 1.618 & O68 & 1.680 & O99 & 1.600 \\
\hline O7 & 1.658 & O38 & 1.492 & O69 & 1.689 & $\mathrm{O} 100$ & 1.558 \\
\hline $\mathrm{O} 8$ & 1.644 & O39 & 1.579 & O70 & 1.685 & O101 & 1.676 \\
\hline O9 & 1.662 & $\mathrm{O} 40$ & 1.685 & O71 & 1.653 & $\mathrm{O} 102$ & 1.740 \\
\hline $\mathrm{O} 10$ & 1.971 & O41 & 2.020 & O72 & 1.987 & O103 & 2.063 \\
\hline O11 & 2.044 & O42 & 2.041 & O73 & 2.106 & O104 & 1.974 \\
\hline $\mathrm{O} 12$ & 2.000 & $\mathrm{O} 43$ & 2.061 & $\mathrm{O} 74$ & 1.954 & O105 & 2.069 \\
\hline O13 & 2.055 & O44 & 1.903 & O75 & 2.053 & O106 & 1.965 \\
\hline $\mathrm{O} 14$ & 2.030 & $\mathrm{O} 45$ & 2.055 & O76 & 2.001 & O107 & 2.141 \\
\hline $\mathrm{O} 15$ & 2.068 & O46 & 1.991 & O77 & 2.107 & O108 & 1.956 \\
\hline O16 & 1.808 & O47 & 1.808 & O78 & 1.697 & O109 & 1.777 \\
\hline O17 & 1.796 & O48 & 1.817 & O79 & 1.841 & $\mathrm{O} 110$ & 1.843 \\
\hline O18 & 1.827 & O49 & 1.795 & O80 & 1.816 & $\mathrm{O} 111$ & 1.825 \\
\hline O19 & 2.040 & O50 & 2.042 & O81 & 2.028 & O112 & 2.102 \\
\hline $\mathrm{O} 20$ & 1.838 & O51 & 1.860 & O82 & 1.913 & O113 & 1.804 \\
\hline $\mathrm{O} 21$ & 1.968 & O52 & 1.893 & O83 & 2.009 & $\mathrm{O} 114$ & 2.002 \\
\hline $\mathrm{O} 22$ & 1.874 & O53 & 1.977 & O84 & 1.928 & $\mathrm{O} 115$ & 1.952 \\
\hline $\mathrm{O} 23$ & 2.044 & O54 & 2.027 & O85 & 1.938 & O116 & 1.946 \\
\hline $\mathrm{O} 24$ & 1.895 & O55 & 1.923 & O86 & 1.830 & O117 & 1.948 \\
\hline $\mathrm{O} 25$ & 1.880 & O56 & 1.774 & O87 & 1.882 & O118 & 1.944 \\
\hline $\mathrm{O} 26$ & 1.667 & O57 & 1.915 & O88 & 1.726 & O119 & 1.896 \\
\hline $\mathrm{O} 27$ & 1.769 & O58 & 1.976 & O89 & 1.488 & O120 & 1.880 \\
\hline $\mathrm{O} 28$ & 1.847 & O59 & 1.842 & O90 & 1.863 & O121 & 1.892 \\
\hline $\mathrm{O} 29$ & 1.924 & O60 & 1.955 & O91 & 1.914 & $\mathrm{O} 122$ & 1.874 \\
\hline O30 & 1.895 & O61 & 1.759 & O92 & 1.807 & O123 & 1.566 \\
\hline O31 & 1.967 & O62 & 2.136 & O93 & 1.853 & $\mathrm{O} 124$ & 1.588 \\
\hline
\end{tabular}


Table S7. BVS values for phosphorous, molybdenum and oxygen of TPP-III•ANQ.

\begin{tabular}{|c|c|c|c|c|c|c|c|}
\hline $\mathrm{P} 1$ & 4.810 & $\mathrm{P} 2$ & 4.630 & P3 & 4.589 & P4 & 4.762 \\
\hline Mo1 & 5.921 & Mo10 & 6.092 & Mo19 & 6.120 & Mo28 & 6.102 \\
\hline Mo2 & 6.120 & Mo11 & 6.191 & Mo20 & 5.937 & Mo29 & 6.193 \\
\hline Mo3 & 6.130 & Mo12 & 6.325 & Mo21 & 6.170 & Mo30 & 5.969 \\
\hline Mo4 & 6.104 & Mo13 & 5.999 & Mo22 & 6.002 & Mo31 & 6.072 \\
\hline Mo5 & 6.090 & Mo14 & 6.117 & Mo23 & 6.082 & Mo32 & 6.111 \\
\hline Mo6 & 5.921 & Mo15 & 6.020 & Mo24 & 6.031 & Mo33 & 6.090 \\
\hline Mo7 & 5.963 & Mo16 & 6.234 & Mo25 & 6.093 & Mo34 & 5.928 \\
\hline Mo8 & 5.987 & Mo17 & 5.969 & Mo26 & 5.993 & Mo35 & 5.963 \\
\hline Mo9 & 5.978 & Mo18 & 5.967 & Mo27 & 5.944 & Mo36 & 6.027 \\
\hline $\mathrm{O} 1$ & 1.694 & $\mathrm{O} 32$ & 1.822 & O63 & 1.857 & O94 & 1.774 \\
\hline $\mathrm{O} 2$ & 1.862 & O33 & 1.812 & O64 & 1.680 & O95 & 1.689 \\
\hline $\mathrm{O} 3$ & 1.842 & O34 & 1.918 & O65 & 1.939 & O96 & 1.712 \\
\hline O4 & 1.862 & O35 & 1.740 & O66 & 1.736 & O97 & 1.788 \\
\hline O5 & 1.764 & O36 & 1.769 & O67 & 1.750 & O98 & 1.852 \\
\hline O6 & 1.676 & O37 & 1.698 & O68 & 1.764 & O99 & 1.803 \\
\hline O7 & 1.759 & O38 & 1.653 & O69 & 1.653 & $\mathrm{O} 100$ & 1.832 \\
\hline O8 & 1.685 & O39 & 1.793 & $\mathrm{O} 70$ & 1.653 & O101 & 1.708 \\
\hline O9 & 1.631 & O40 & 1.689 & O71 & 1.658 & O102 & 1.644 \\
\hline $\mathrm{O} 10$ & 1.965 & O41 & 2.044 & $\mathrm{O} 72$ & 1.949 & $\mathrm{O} 103$ & 2.063 \\
\hline O11 & 2.094 & O42 & 2.068 & O73 & 2.054 & O104 & 2.098 \\
\hline $\mathrm{O} 12$ & 1.990 & O43 & 1.925 & O74 & 1.970 & O105 & 1.928 \\
\hline O13 & 2.064 & O44 & 2.046 & O75 & 2.055 & O106 & 2.088 \\
\hline $\mathrm{O} 14$ & 1.949 & O45 & 1.997 & O76 & 1.970 & O107 & 1.929 \\
\hline O15 & 2.037 & O46 & 2.081 & O77 & 2.096 & O108 & 2.076 \\
\hline O16 & 1.747 & O47 & 1.745 & O78 & 1.781 & O109 & 2.017 \\
\hline O17 & 1.816 & O48 & 1.758 & O79 & 1.788 & $\mathrm{O} 110$ & 1.862 \\
\hline O18 & 1.906 & O49 & 1.796 & O80 & 1.771 & O111 & 2.075 \\
\hline O19 & 1.966 & O50 & 2.020 & O81 & 1.959 & $\mathrm{O} 112$ & 1.827 \\
\hline $\mathrm{O} 20$ & 1.886 & O51 & 1.872 & O82 & 1.805 & $\mathrm{O} 113$ & 1.946 \\
\hline $\mathrm{O} 21$ & 1.941 & O52 & 2.070 & O83 & 1.952 & $\mathrm{O} 114$ & 1.850 \\
\hline $\mathrm{O} 22$ & 1.854 & O53 & 1.874 & O84 & 1.926 & $\mathrm{O} 115$ & 1.778 \\
\hline $\mathrm{O} 23$ & 1.978 & O54 & 2.046 & O85 & 2.024 & O116 & 1.825 \\
\hline $\mathrm{O} 24$ & 1.854 & O55 & 1.910 & O86 & 1.918 & $\mathrm{O} 117$ & 1.818 \\
\hline $\mathrm{O} 25$ & 1.910 & O56 & 1.923 & O87 & 1.893 & $\mathrm{O} 118$ & 1.917 \\
\hline $\mathrm{O} 26$ & 1.644 & O57 & 1.842 & O88 & 1.812 & O119 & 1.726 \\
\hline $\mathrm{O} 27$ & 1.822 & O58 & 1.750 & O89 & 1.754 & O120 & 1.754 \\
\hline $\mathrm{O} 28$ & 1.694 & O59 & 1.667 & O90 & 1.698 & O121 & 1.759 \\
\hline $\mathrm{O} 29$ & 1.923 & O60 & 1.957 & O91 & 1.919 & $\mathrm{O} 122$ & 1.908 \\
\hline O30 & 1.872 & O61 & 1.932 & O92 & 1.963 & $\mathrm{O} 123$ & 1.945 \\
\hline $\mathrm{O} 31$ & 2.006 & O62 & 1.976 & O93 & 1.880 & $\mathrm{O} 124$ & 1.948 \\
\hline
\end{tabular}


Table S8. Mo-N bond lengths ( $\AA$ ) for TBA-I, TPP-I, TBA-II and TPP-III.

\begin{tabular}{|c|c|c|c|c|c|c|c|}
\hline \multicolumn{2}{|c|}{ TBA-I } & \multicolumn{2}{|c|}{ TPP-I } & \multicolumn{2}{|c|}{ TBA-II } & \multicolumn{2}{|c|}{ TPP-III } \\
\hline Mo2-N1 & 2.313 & Mo2-N1 & 2.333 & Mo2-N1 & 2.326 & Mo1-N1 & 2.306 \\
\hline Mo4-N2 & 2.303 & & & Mo4-N2 & 2.319 & Mo3-N2 & 2.330 \\
\hline Mo6-N3 & 2.311 & & & Mo6-N3 & 2.330 & Mo5-N3 & 2.342 \\
\hline & & & & & & Mo10-N4 & 2.305 \\
\hline & & & & & & Mo12-N5 & 2.331 \\
\hline & & & & & & Mo14-N6 & 2.293 \\
\hline & & & & & & Mo19-N7 & 2.330 \\
\hline & & & & & & Mo21-N8 & 2.308 \\
\hline & & & & & & Mo23-N9 & 2.315 \\
\hline & & & & & & Mo28-N10 & 2.360 \\
\hline & & & & & & Mo30-N11 & 2.289 \\
\hline & & & & & & Mo32-N12 & 2.294 \\
\hline
\end{tabular}


a)

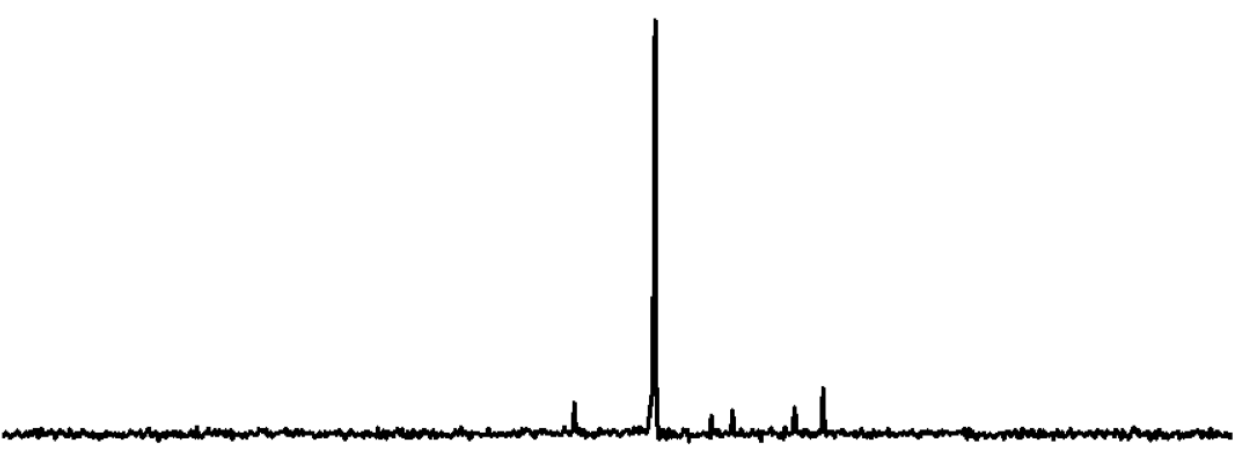

b)
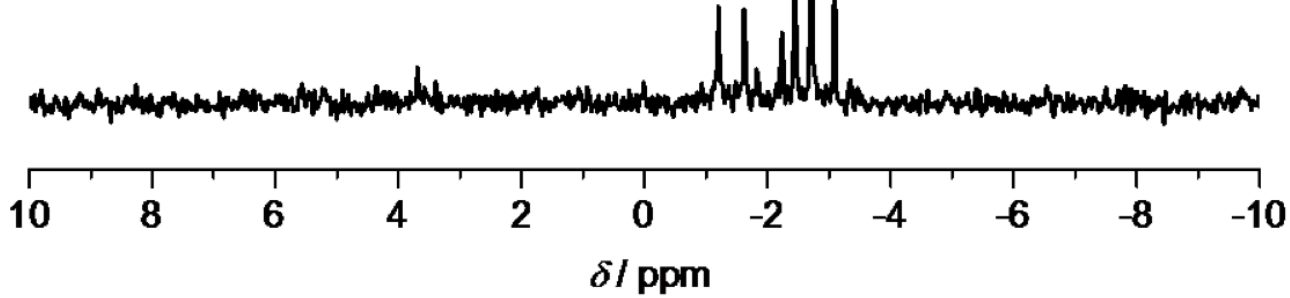

Figure S1. ${ }^{31} \mathrm{P}$ NMR spectra of (a) as-prepared $\mathrm{TBA}_{3} \mathrm{H}_{6}\left[\mathrm{~A}-\alpha-\mathrm{PMo}_{9} \mathrm{O}_{34}\right]$ in dimethyl sulfoxide- $d_{6}$, and (b) as-prepared $\mathrm{TBA}_{3} \mathrm{H}_{6}\left[\mathrm{~A}-\alpha-\mathrm{PMo}_{9} \mathrm{O}_{34}\right]$ in acetonitrile- $d_{3}$. 


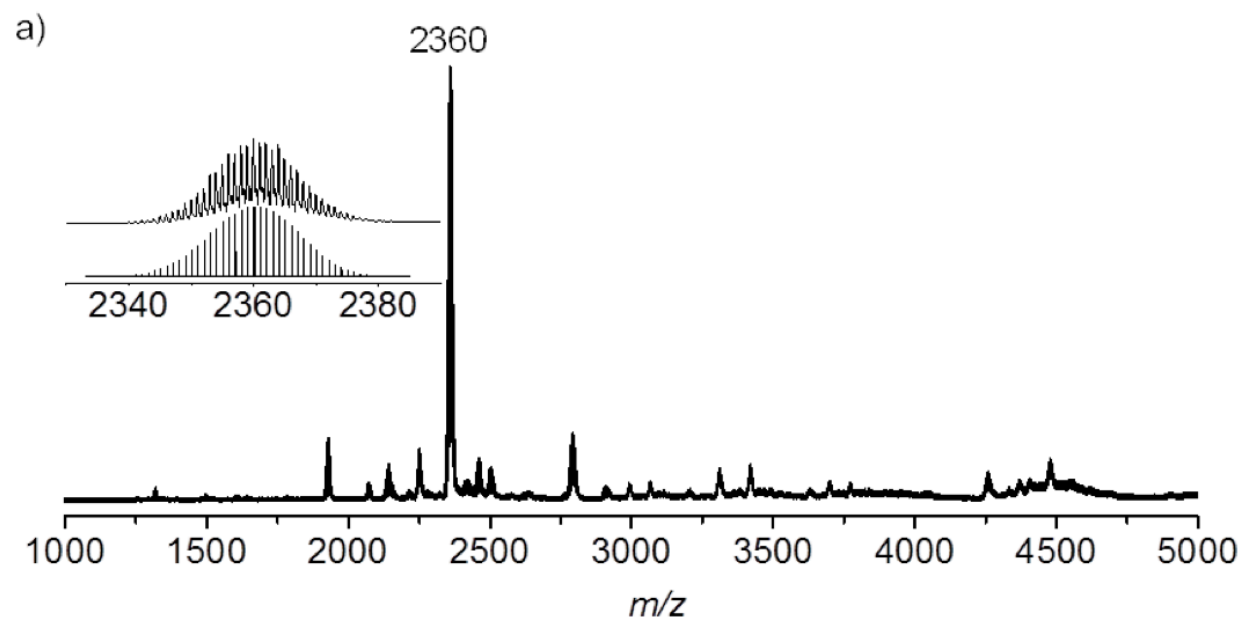

b)

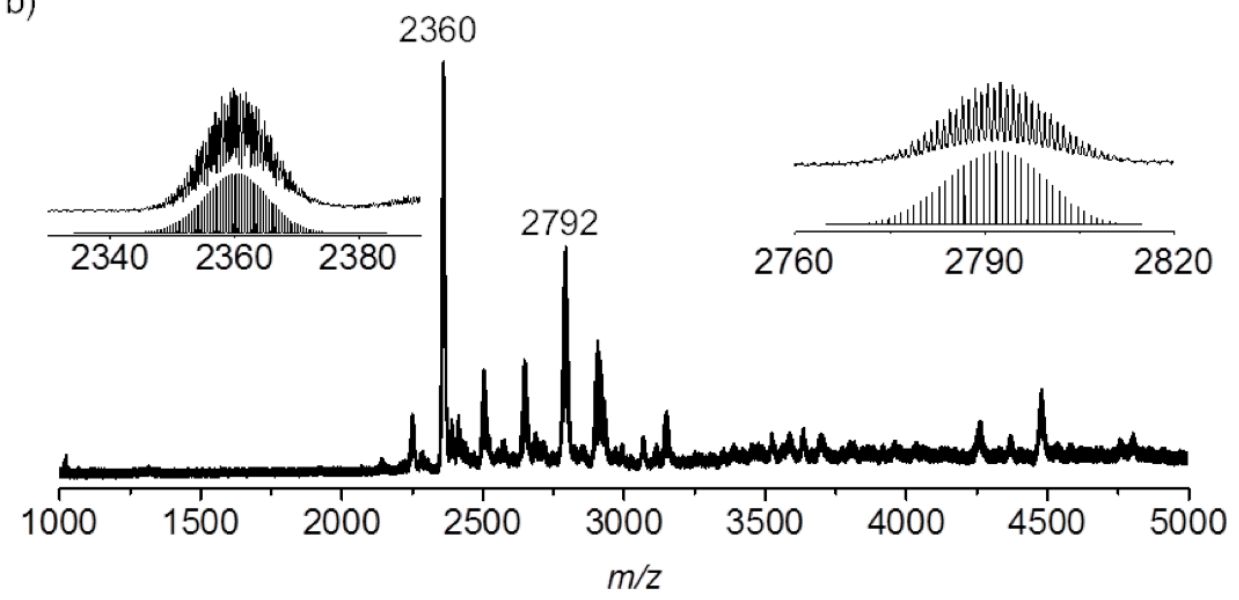

c)

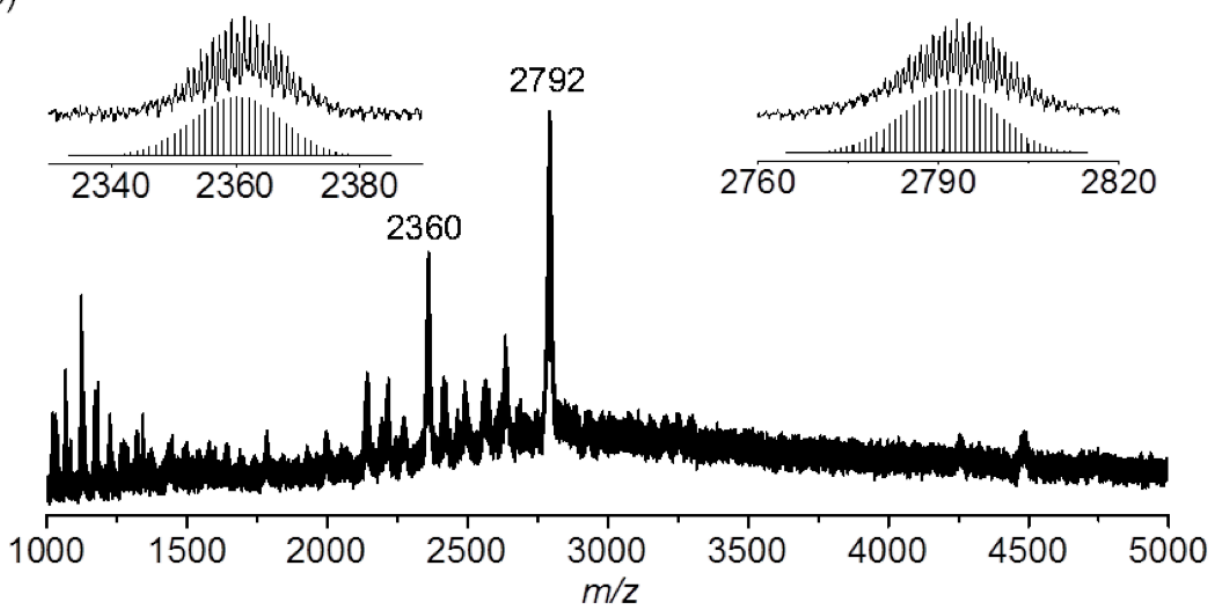

Figure S2. Positive-ion CSI mass spectra of $\mathrm{TBA}_{3} \mathrm{H}_{6}\left[\mathrm{~A}-\alpha-\mathrm{PMo}_{9} \mathrm{O}_{34}\right]$ a) as-prepared sample in acetonitrile, b) sample after $5 \mathrm{~min}$, and c) as-prepared sample in methanol. Insets: a) a spectrum in the range of $\mathrm{m} / \mathrm{z}$ 2340-2380, and a simulated pattern for $\left[\mathrm{TBA}_{4} \mathrm{PMo}_{9} \mathrm{O}_{31}\right]^{+}(\mathrm{m} / z$ 2360); b) spectra in the range of $\mathrm{m} / z$ 23302390 and 2760-2820, and simulated patterns for $\left[\mathrm{TBA}_{8} \mathrm{P}_{2} \mathrm{Mo}_{18} \mathrm{O}_{62}\right]^{2+}\left(\mathrm{m} / z\right.$ 2360) and $\left[\mathrm{TBA}_{4} \mathrm{PMo}_{12} \mathrm{O}_{40}\right]^{+}$ $(\mathrm{m} / \mathrm{z} 2792)$; c) spectra in the range of $\mathrm{m} / \mathrm{z} 2330-2390$ and 2760-2820, and simulated patterns for $\left[\mathrm{TBA}_{4} \mathrm{PMo}_{9} \mathrm{O}_{31}\right]^{+}(m / z 2360)$, and $\left[\mathrm{TBA}_{4} \mathrm{PMo}_{12} \mathrm{O}_{40}\right]^{+}(m / z 2792)$. 
a)

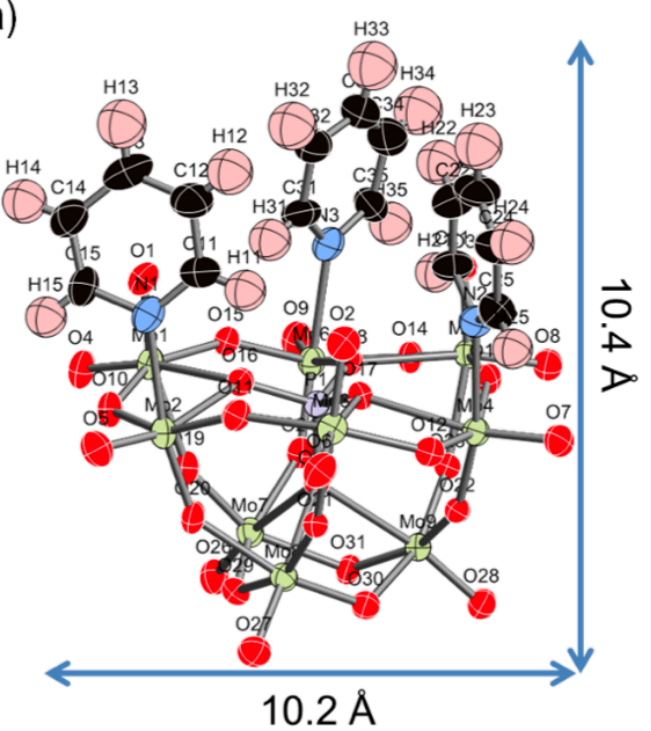

b)

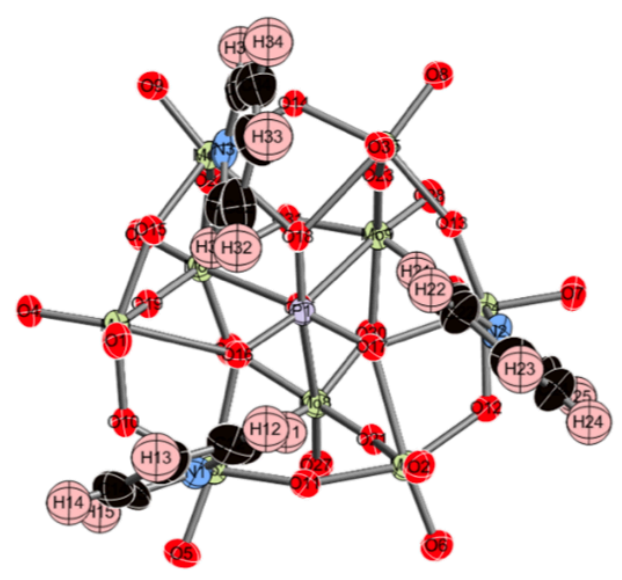

Figure S3. Thermal ellipsoid plots representation (50\% probability level) of the anion part of TBA-I (side view, a; top view, $b$ ).

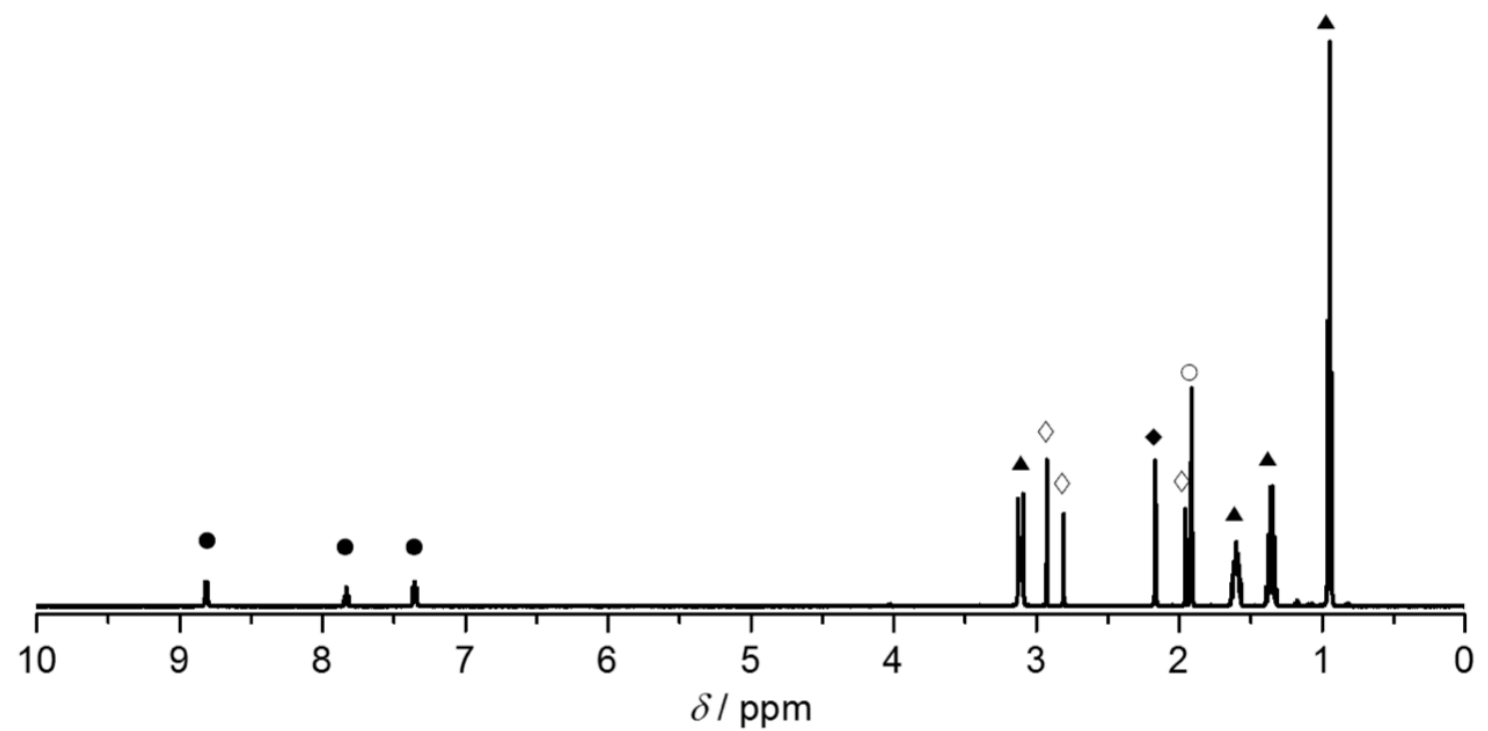

Figure S4. ${ }^{1} \mathrm{H}$ NMR spectrum of TBA-I in acetonitrile- $d_{3}(\boldsymbol{O}$, pyridyl ligand; $\boldsymbol{\Delta}$, TBA; $\diamond$, DMA; $\bigcirc$, acetonitrile; $\diamond$, water). 


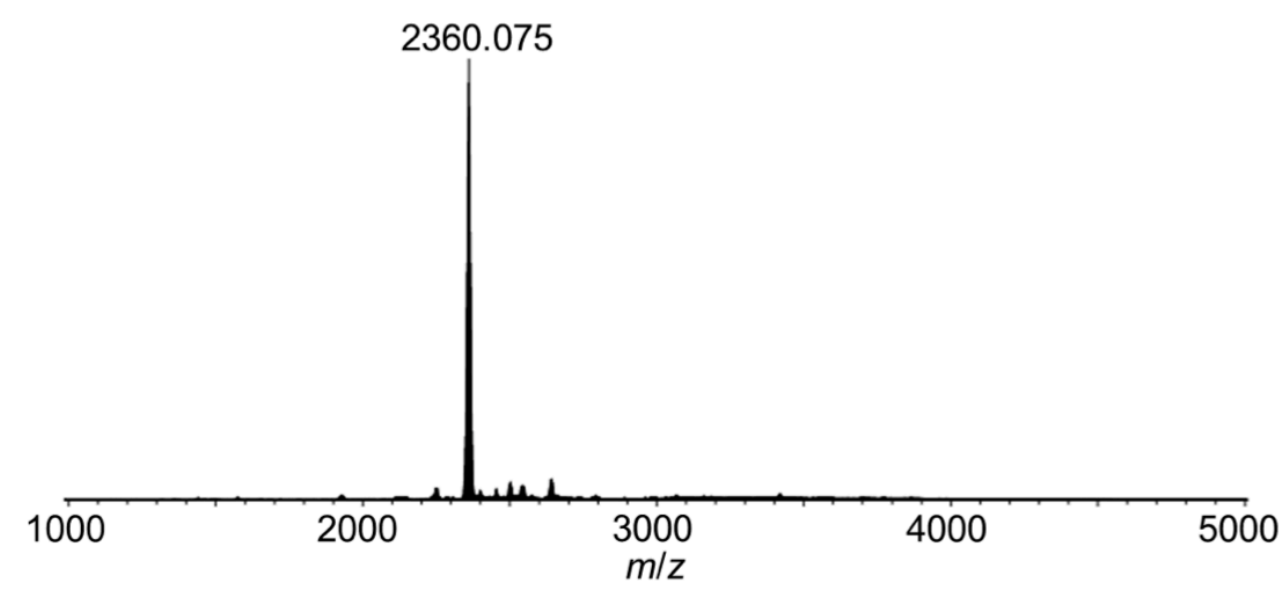

Figure S5. Positive-ion ESI mass spectra of TBA-I in acetonitrile.

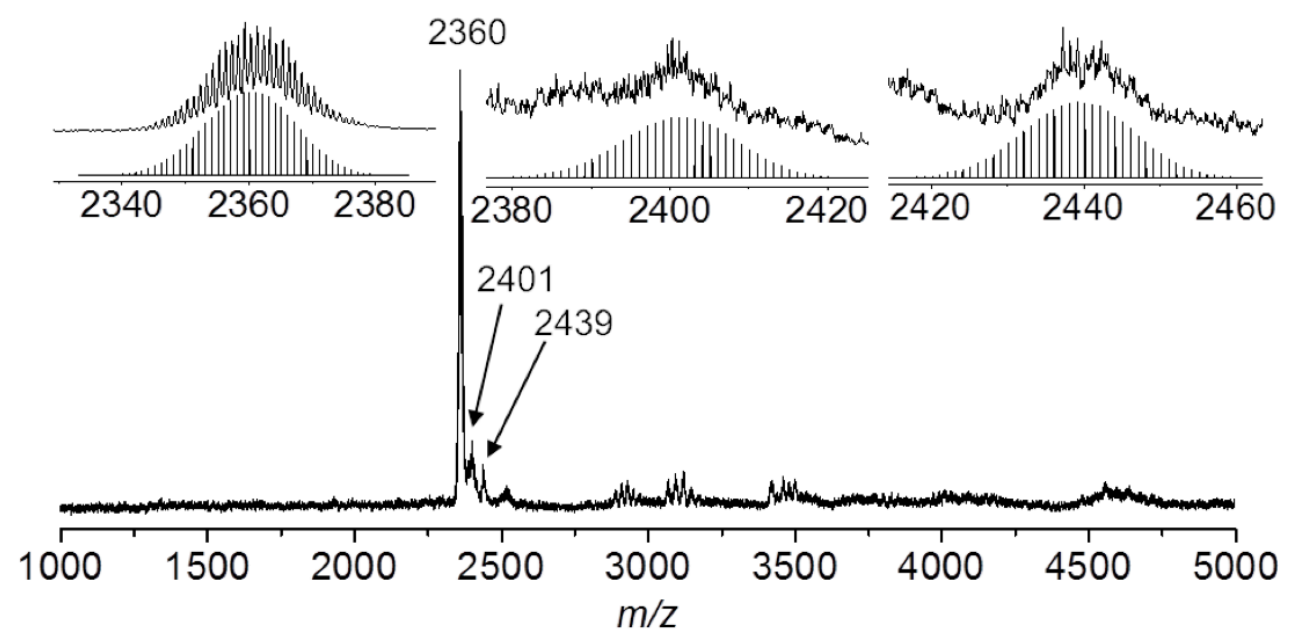

Figure S6. Positive-ion CSI mass spectra of TBA-I in a mixture of acetonitrile and pyridine $(9 / 1, \mathrm{v} / \mathrm{v})$. Insets: spectra in the range of $m / z 2375-2425,2415-2465$, and 2490-2545, and simulated patterns for $\left[\mathrm{TBA}_{4} \mathrm{PMo}_{9} \mathrm{O}_{31}\right]^{+}(\mathrm{m} / z 2360),\left[\mathrm{TBA}_{4} \mathrm{PMo}_{9} \mathrm{O}_{31}\left(\mathrm{CH}_{3} \mathrm{CN}\right)\right]^{+}(\mathrm{m} / z 2401)$, and $\left[\mathrm{TBA}_{4} \mathrm{PMo}_{9} \mathrm{O}_{31}\left(\mathrm{C}_{5} \mathrm{H}_{5} \mathrm{~N}\right)\right]^{+}(\mathrm{m} / z$ 2439). 
a)

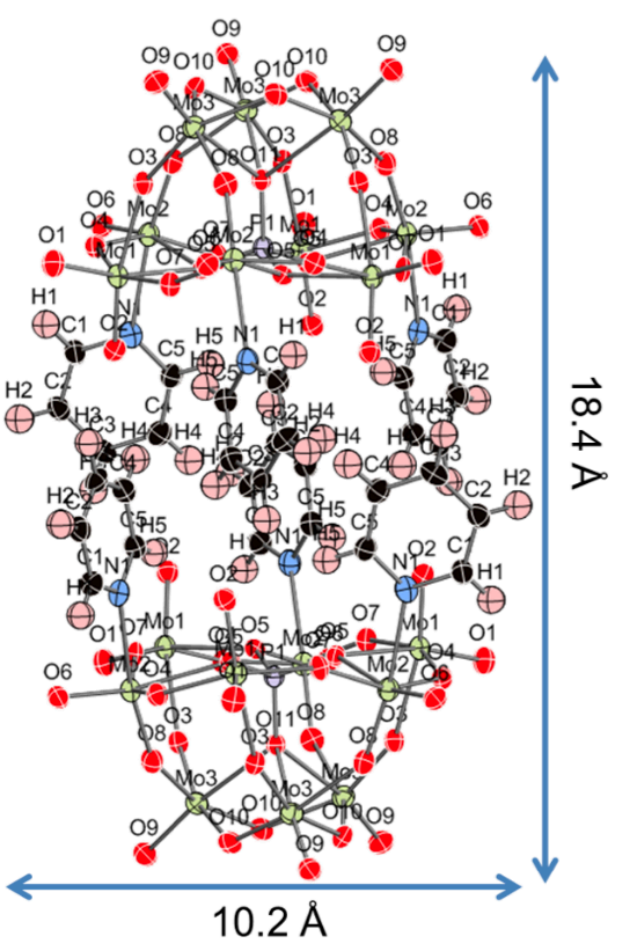

b)

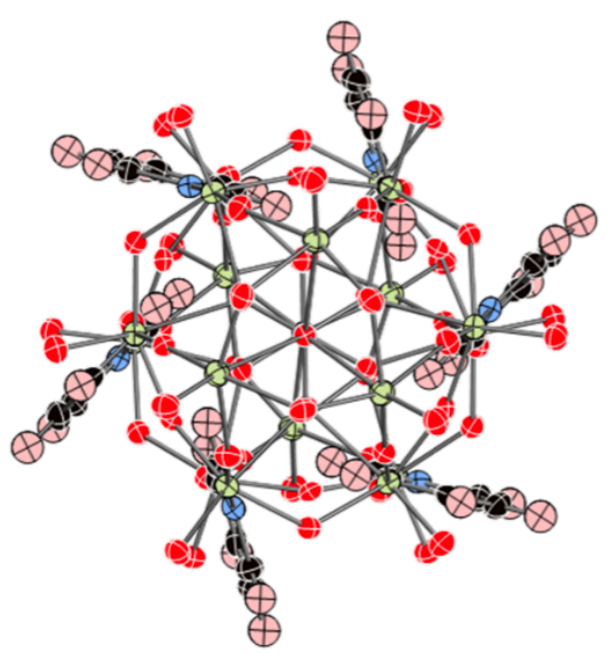

Figure S7. Thermal ellipsoid plots representation (50\% probability level) of the anion parts of TPP-I (side view, a; top view, b). Two anions were observed.

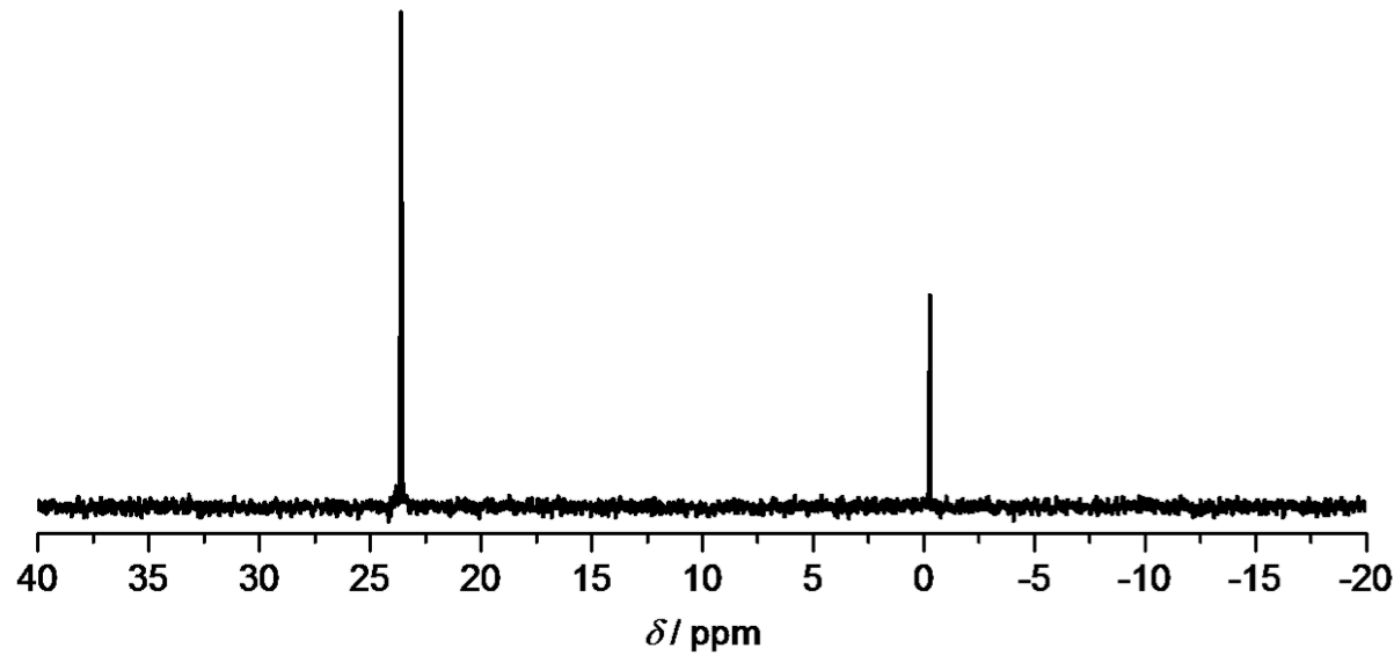

Figure S8. ${ }^{31} \mathrm{P}$ NMR spectrum of TPP-I in acetonitrile- $d_{3}$. 


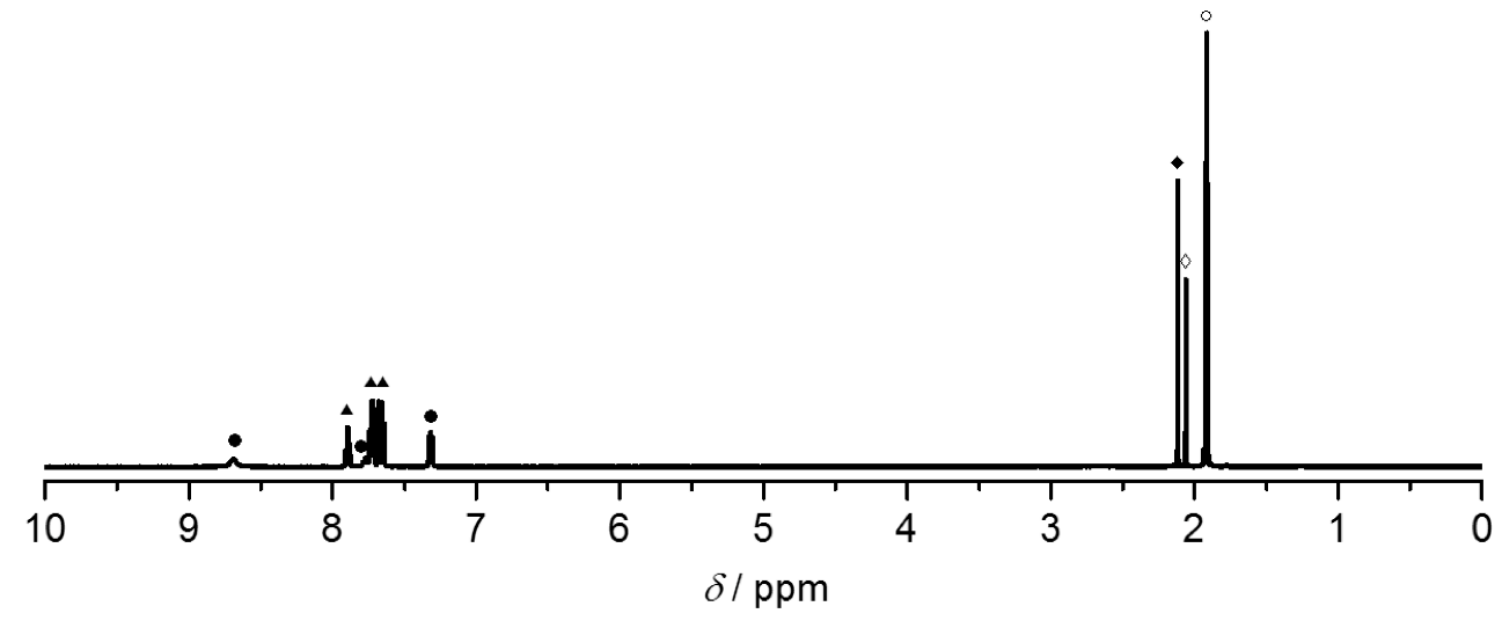

Figure S9. ${ }^{1} \mathrm{H}$ NMR spectrum of TPP-I in acetonitrile- $d_{3}$; $\boldsymbol{\ominus}$, pyridyl ligand; $\boldsymbol{\Delta}$, TPP; $\diamond$, water; $\diamond$, acetone; $\bigcirc$, acetonitrile).

a)

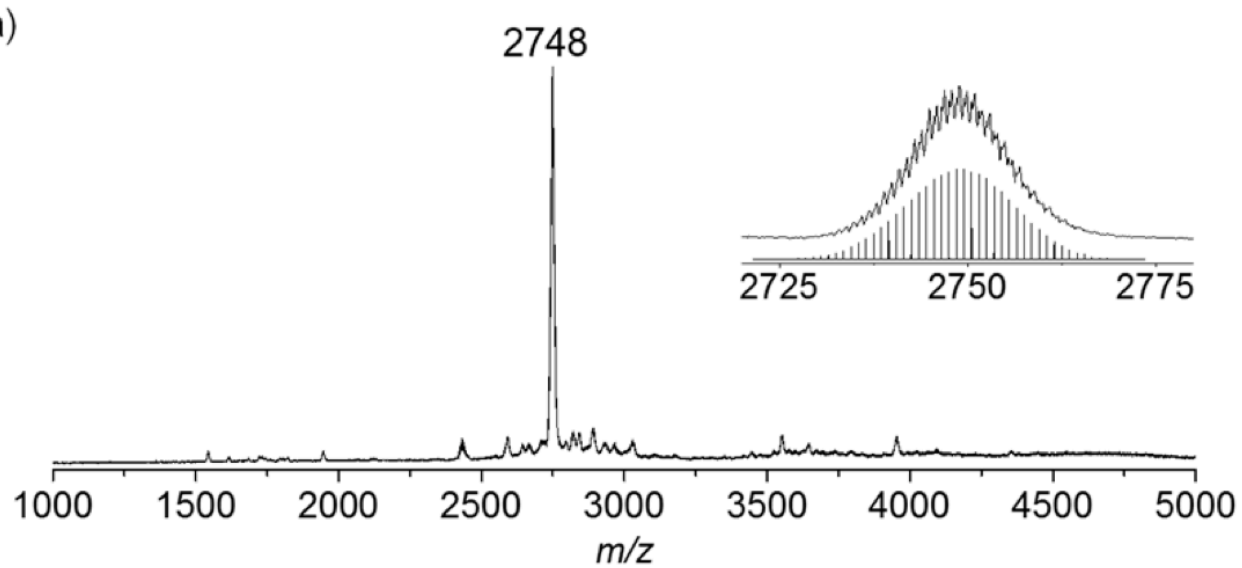

b)

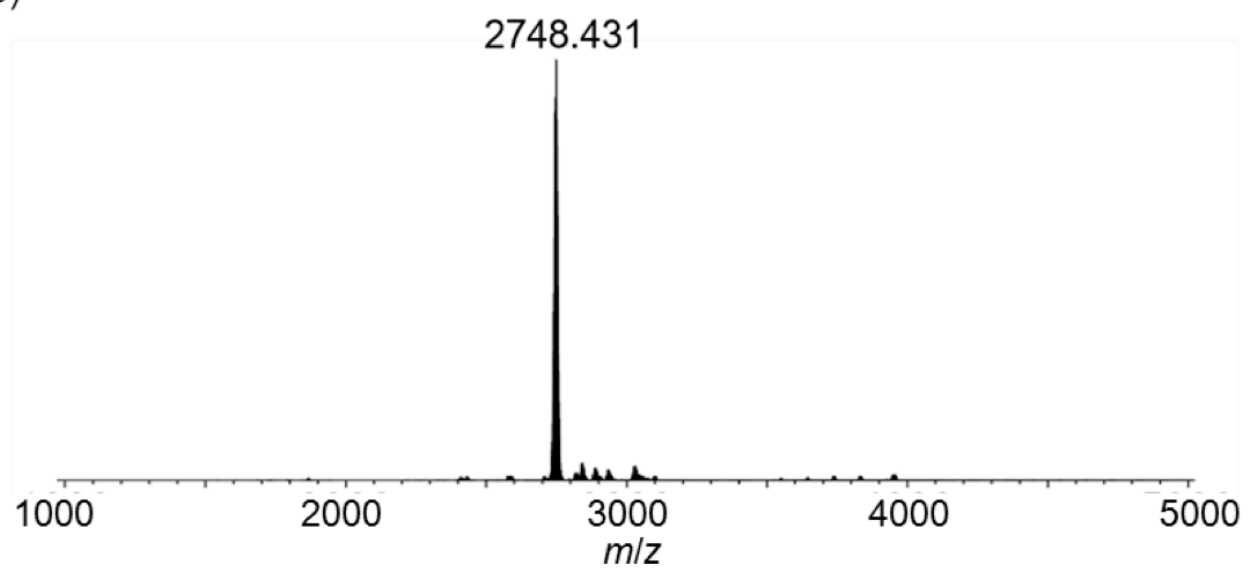

Figure S10. a) Positive-ion CSI mass spectrum and b) positive-ion ESI mass spectrum of TPP-I in acetonitrile. Insets: a spectrum in the range of $\mathrm{m} / \mathrm{z}$ 2720-2780, and simulated patterns for $\left[\mathrm{TPP}_{4} \mathrm{PMo}_{9} \mathrm{O}_{31}\right]^{+}(\mathrm{m} / \mathrm{z} 2748.493)$. 
a)

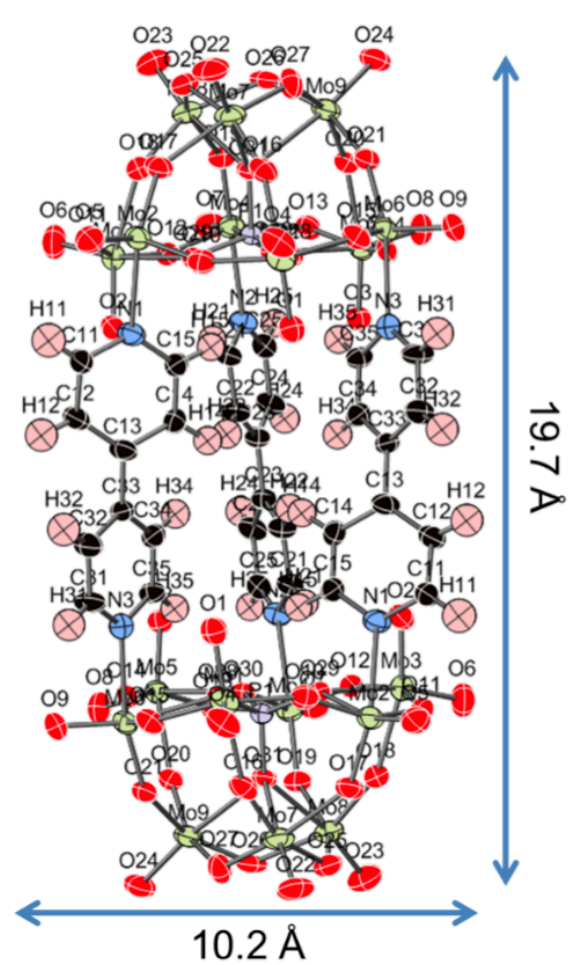

b)

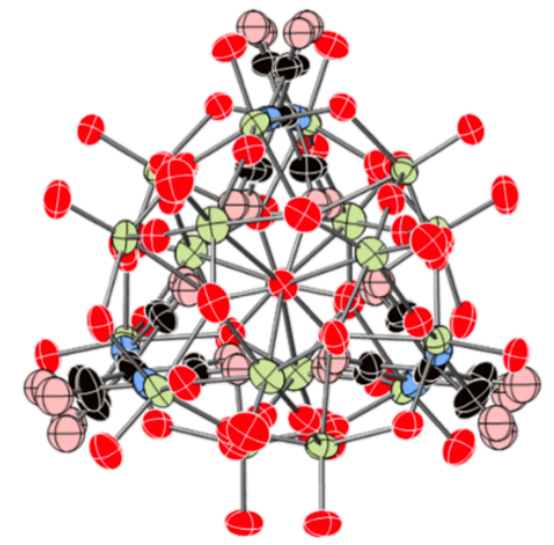

Figure S11. Thermal ellipsoid plots representation (50\% probability level) of the anion part of TBA-II (side view, a; top view, b). 
a)

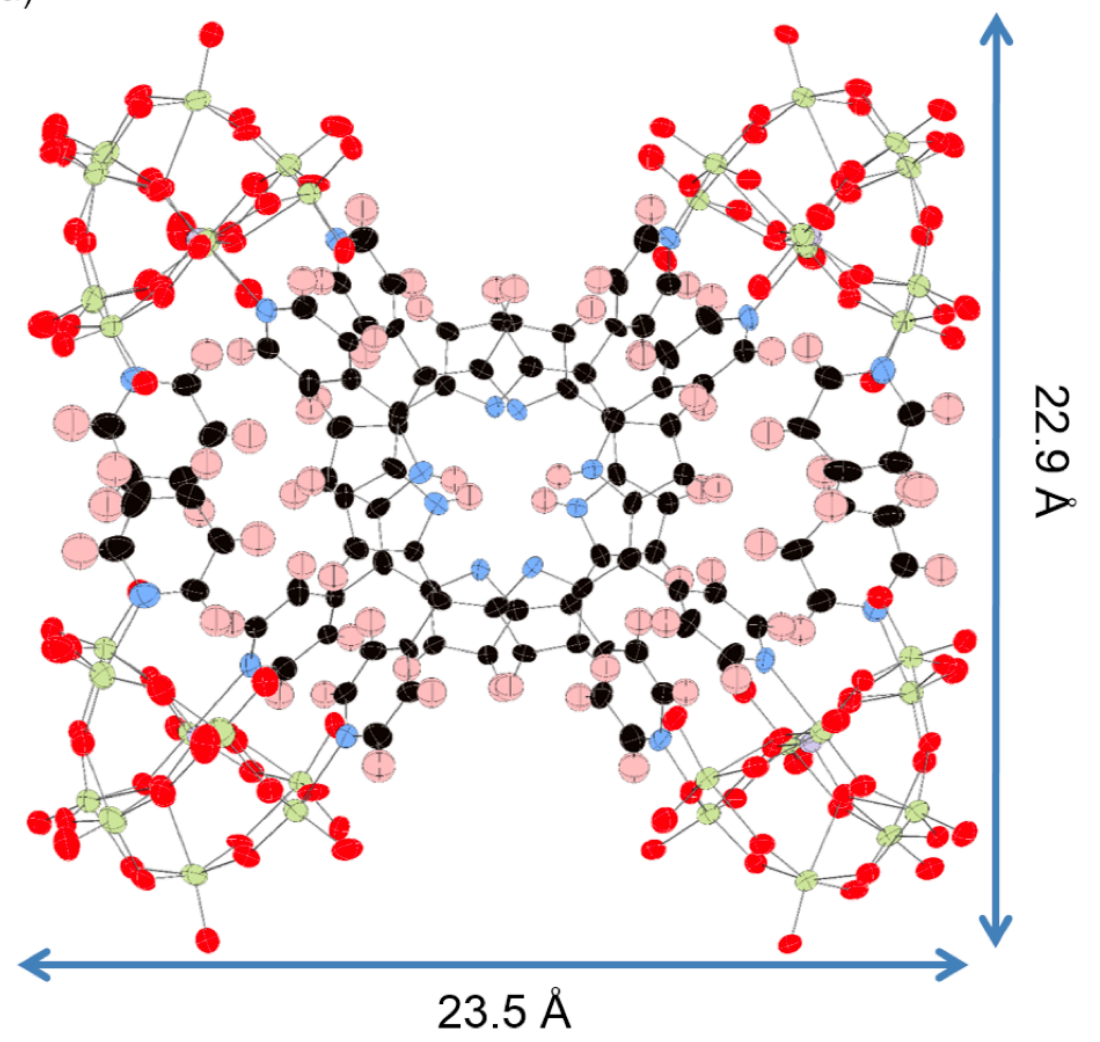

b)

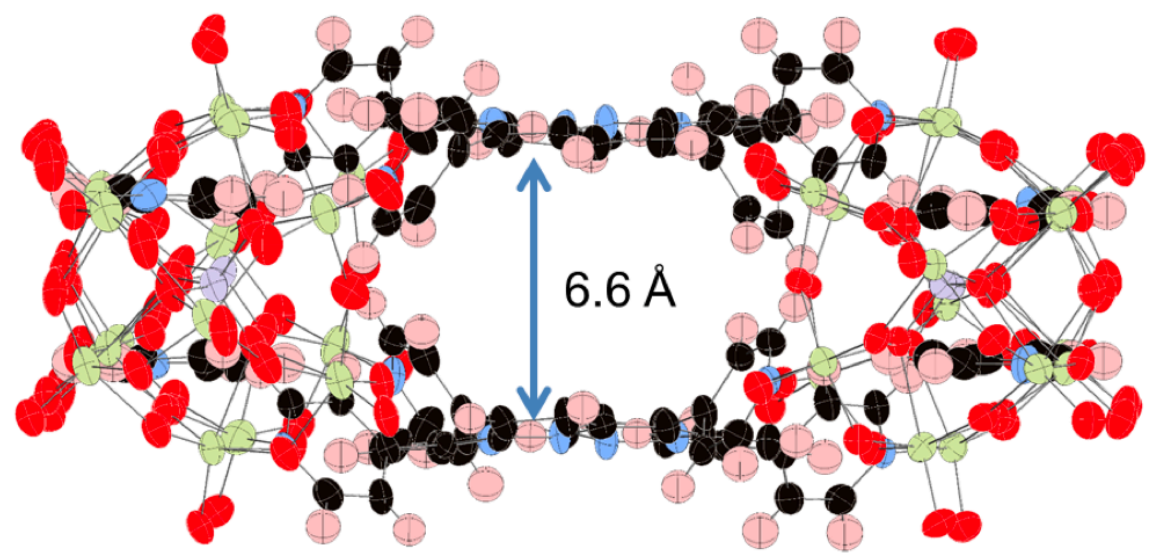

Figure S12. Thermal ellipsoid plots representation (50\% probability level) of the anion part of TPP-III (top view, a; side view, b). 


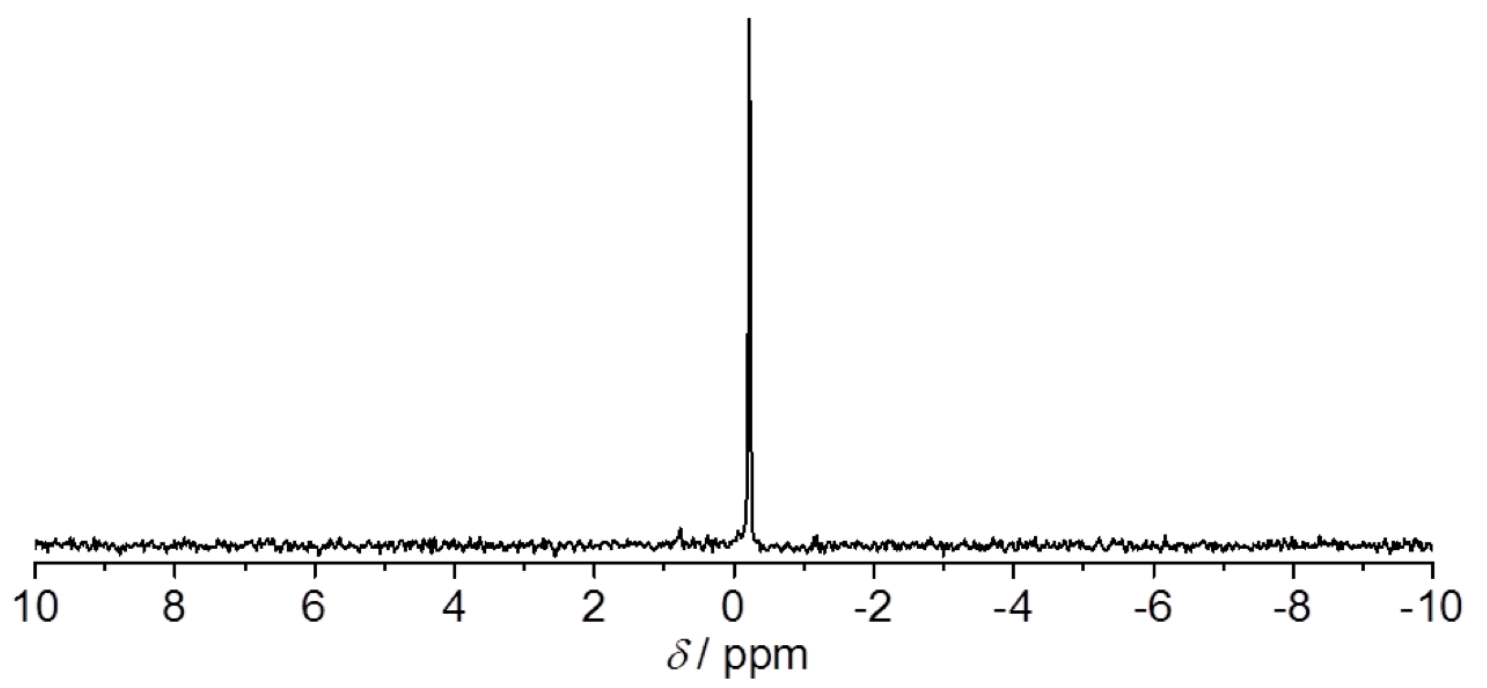

Figure S13. ${ }^{31} \mathrm{P}$ NMR spectrum of TBA-III in acetonitrile- $d_{3}$. 
a)

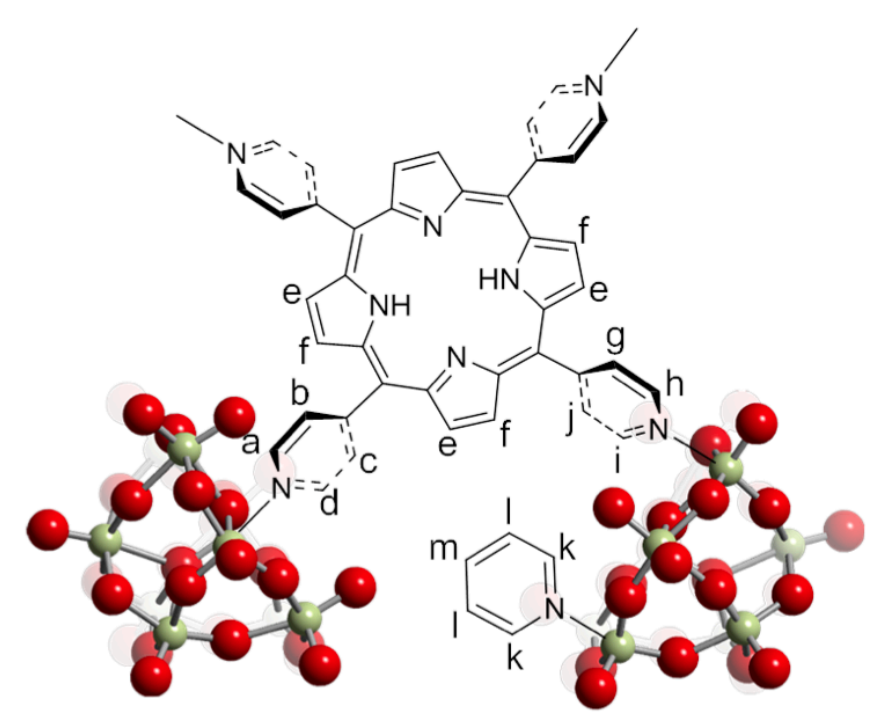

b)

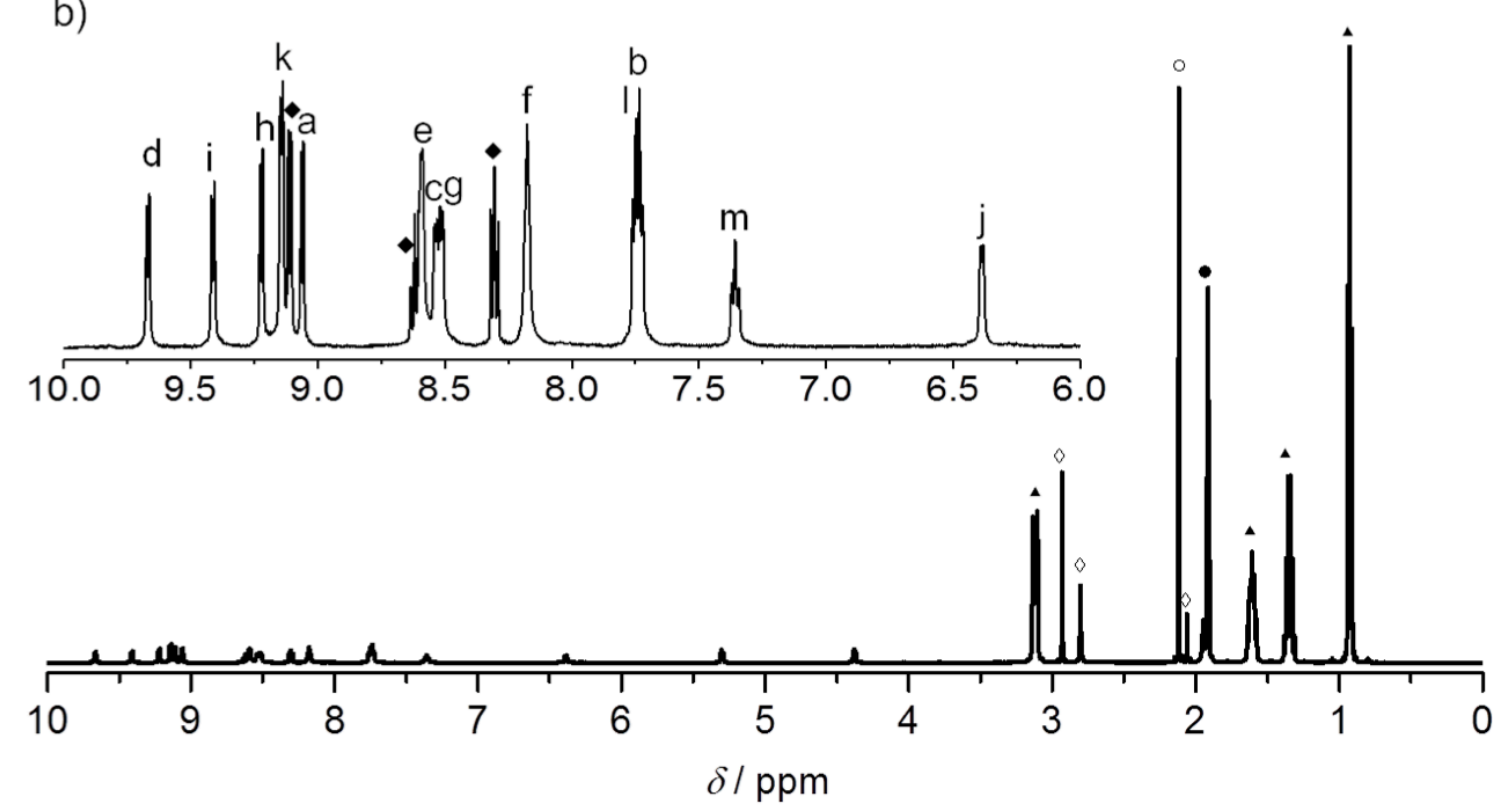

Figure S14. a) Partial ball-stick presentation of the structure of III. Spheres: P purple, Mo green, C black, H pink, N light blue; b) ${ }^{1} \mathrm{H}$ NMR spectrum of TBA-III in acetonitrile- $d_{3}(\boldsymbol{\Delta}$, TBA; $\diamond$, DMA; pyridine; $\bigcirc$, water; $\boldsymbol{O}$, acetonitrile). 


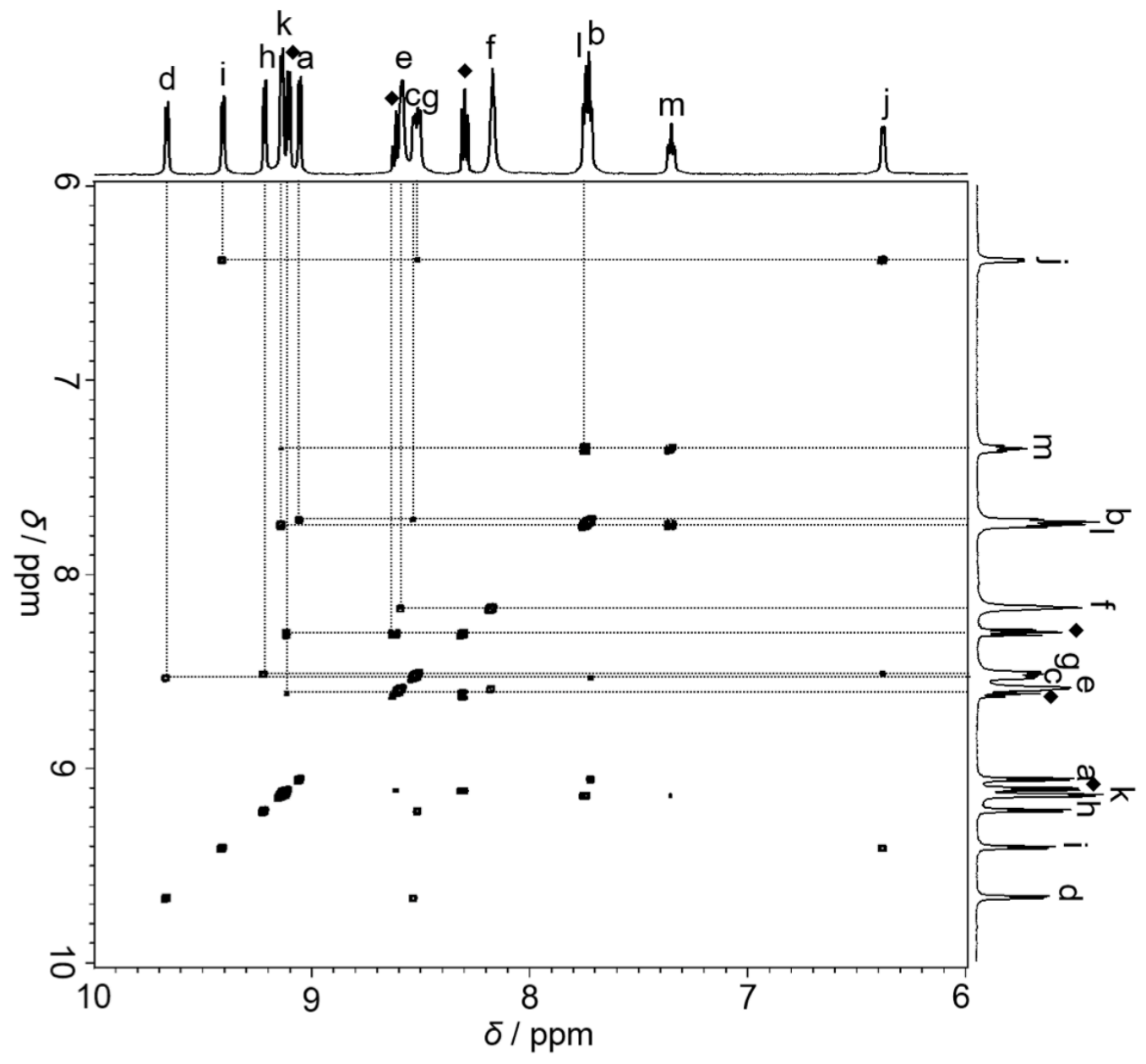

Figure S15. ${ }^{1} \mathrm{H}-{ }^{1} \mathrm{H}$ COSY NMR spectrum of TBA-III in acetonitrile- $d_{3}(\diamond$, pyridine). 


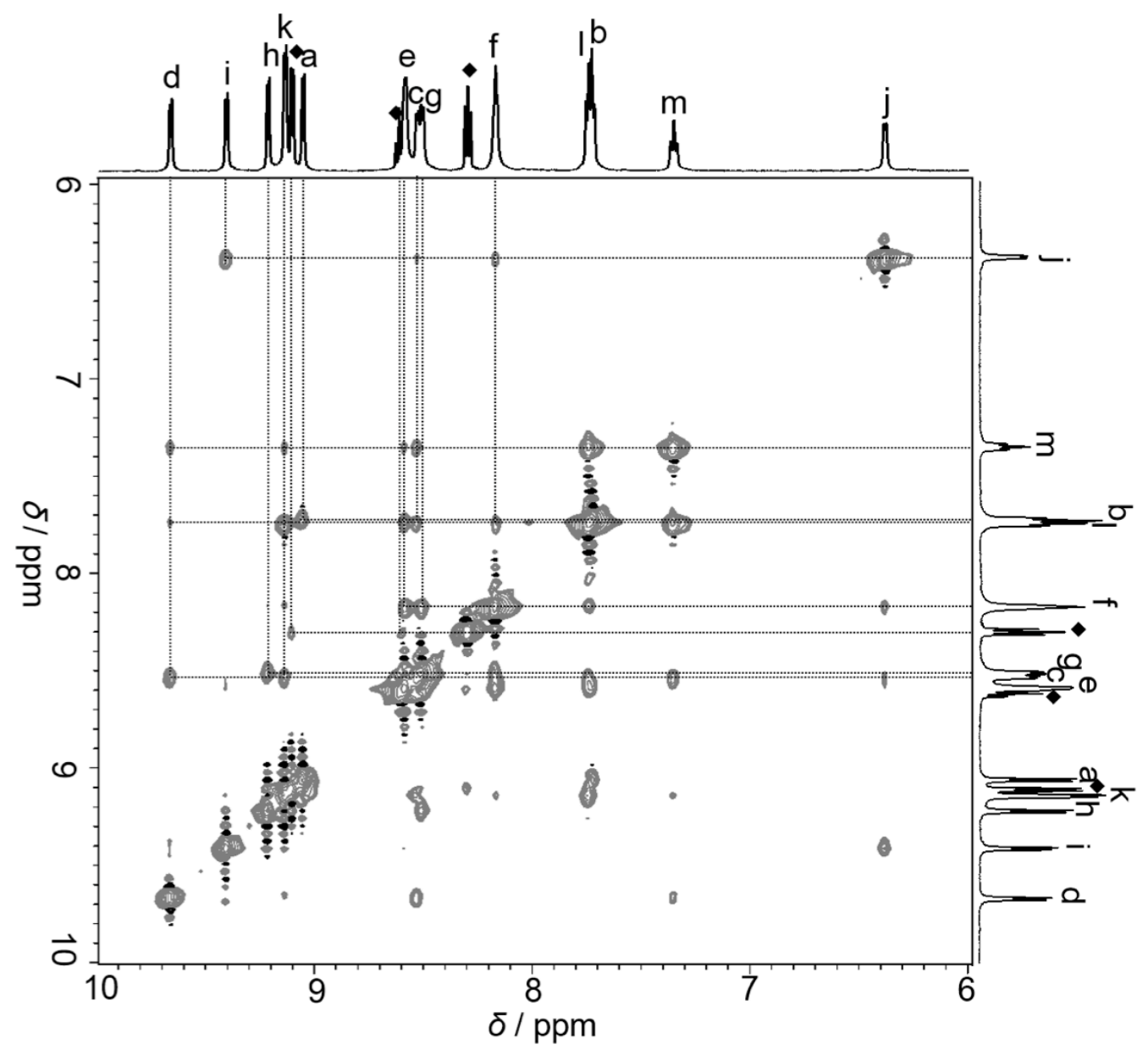

Figure S16. ${ }^{1} \mathrm{H}-{ }^{1} \mathrm{H}$ NOESY NMR spectrum of TBA-III in acetonitrile- $d_{3}(\diamond$, pyridine).

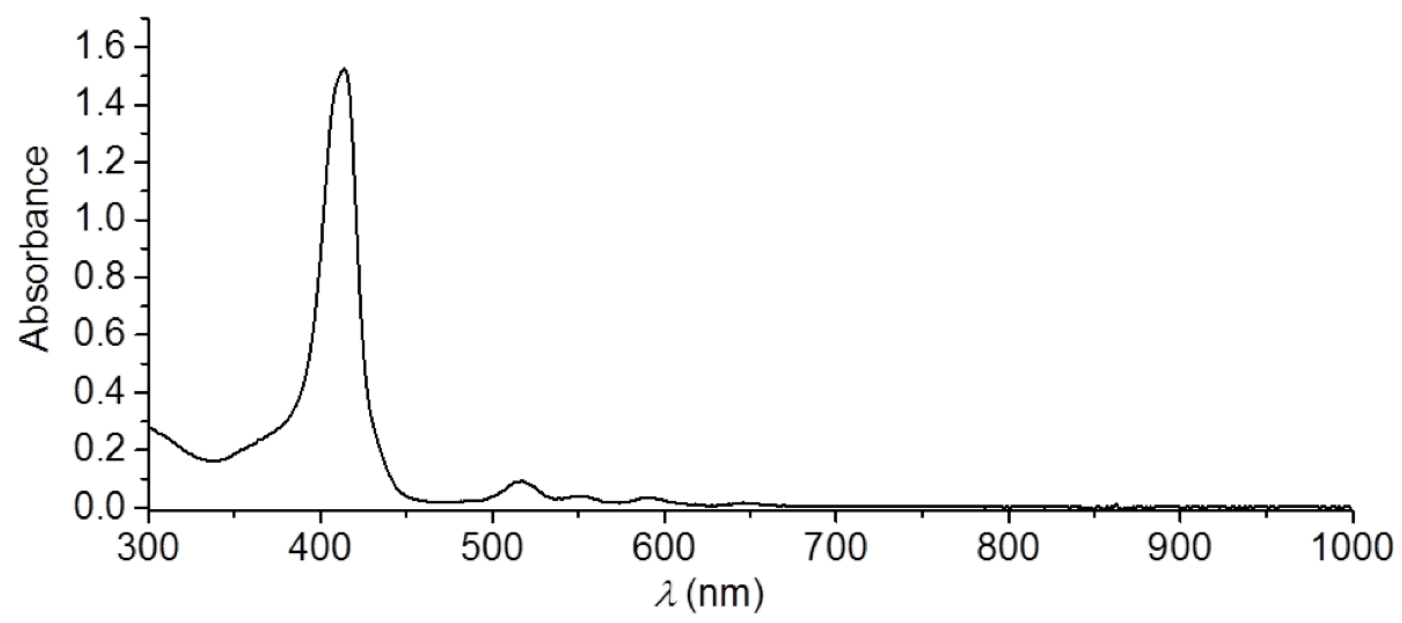

Figure S17. UV-Vis spectrum of TBA-III $(0.0025 \mathrm{mM})$ in acetonitrile. 
a)

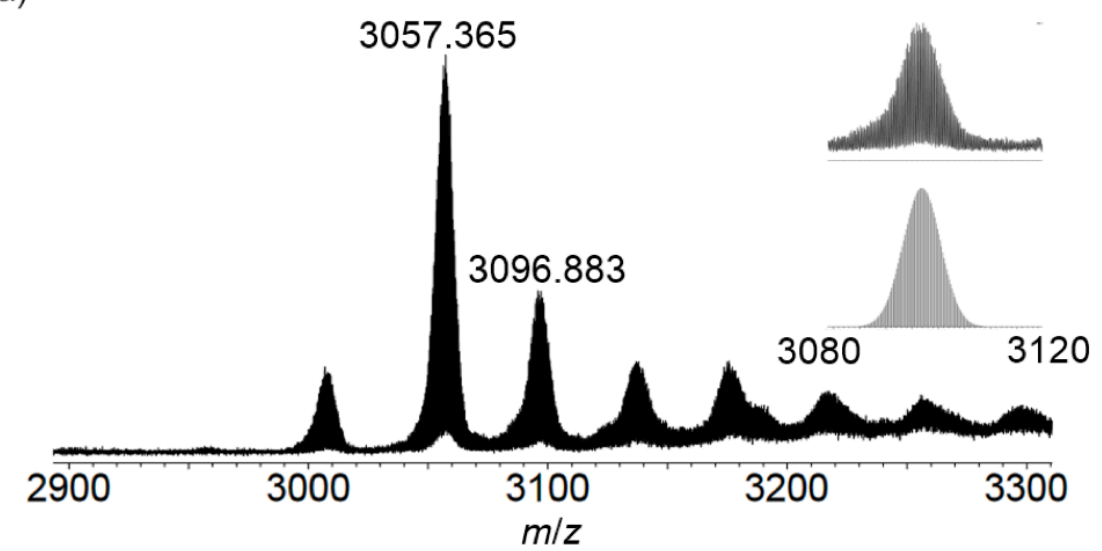

b)

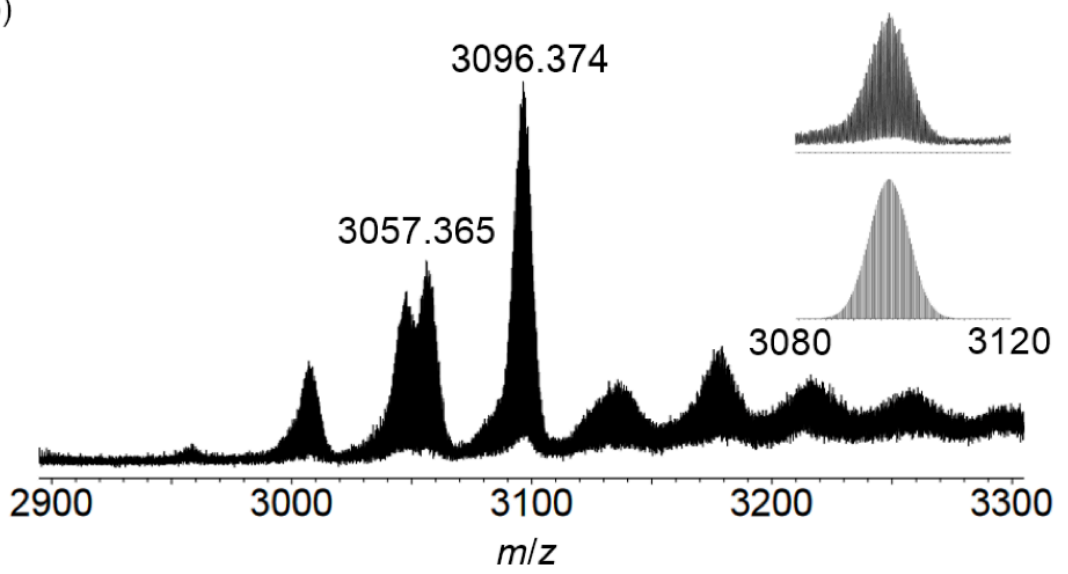

c)

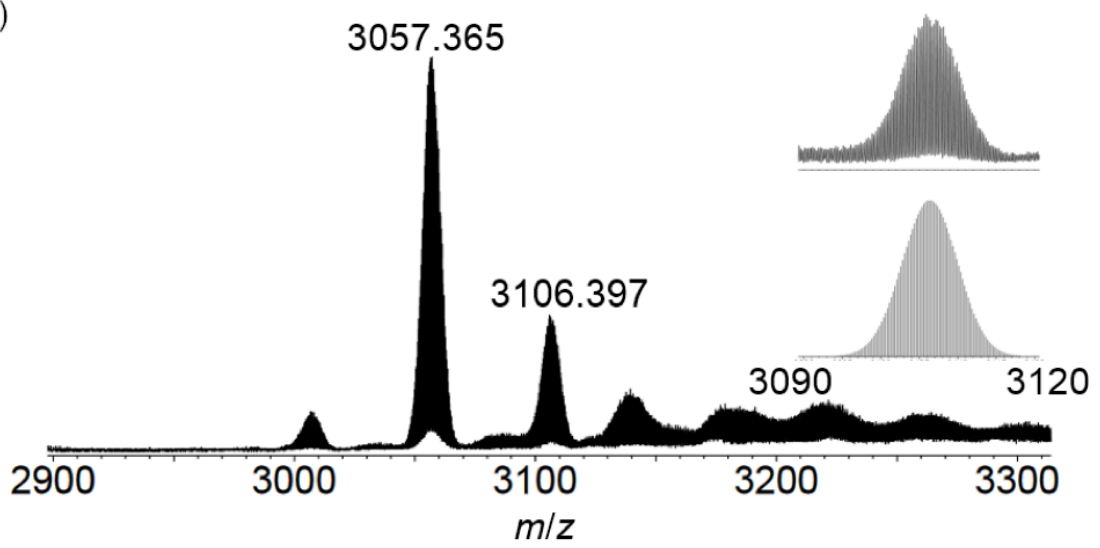

Figure S18. ESI mass spectra of TPP-III in acetonitrile in the presence of guest molecules (10 equiv with respect to III): a) 1,4-dihydroxynaphthalene (G1), b) 1,4-naphthoquinone (G2), and c) 1,8-naphthalic anhydride (G3). Insets: a) spectrum in the range of $\mathrm{m} / \mathrm{z} 3080-3120$, and simulated patterns for $\left[\mathrm{TPP}_{16}\left(\mathrm{PMo}_{9} \mathrm{O}_{31}\right)_{4}(\mathbf{t p y p})_{2} \cdot \mathbf{G 1}\right]^{4+}(\mathrm{m} / \mathrm{z}$ 3096.867); b) spectrum in the range of $\mathrm{m} / \mathrm{z}$ 3080-3120, and simulated patterns for $\left[\mathrm{TPP}_{16}\left(\mathrm{PMo}_{9} \mathrm{O}_{31}\right)_{4}(\mathbf{t p y p})_{2} \cdot \mathbf{G} 2\right]^{4+}(\mathrm{m} / \mathrm{z} 3096.363)$; c) spectrum in the range of $\mathrm{m} / z$ 3090-3120, and simulated patterns for $\left[\mathrm{TPP}_{16}\left(\mathrm{PMo}_{9} \mathrm{O}_{31}\right)_{4}(\mathbf{t p y p})_{2} \cdot \mathbf{G} 3\right]^{4+}(\mathrm{m} / z$ 3106.362). 

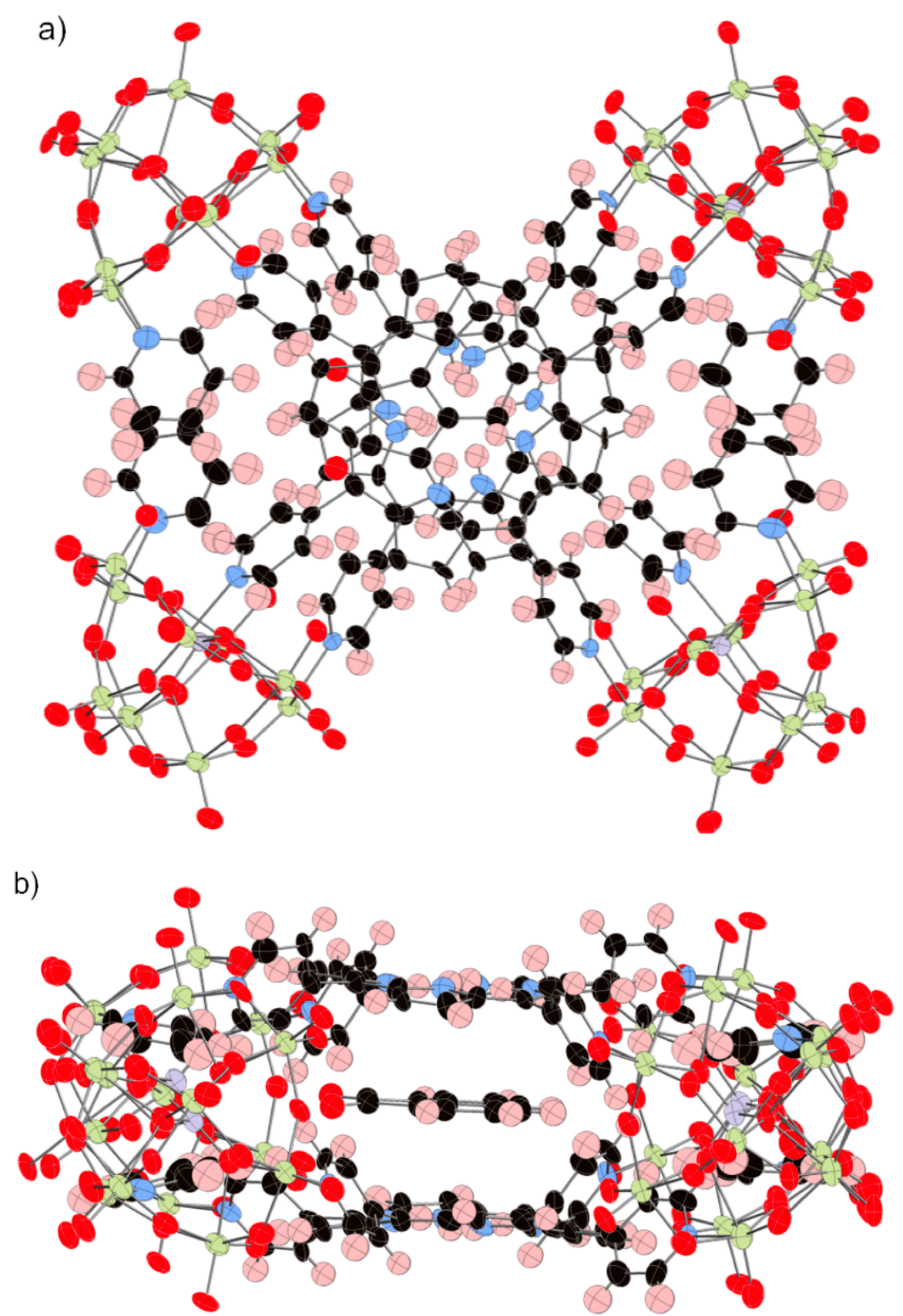

Figure S19. Thermal ellipsoid plots representation (50\% probability level) of the anion part of TPP-III•ANQ (top view, a; side view, b). 


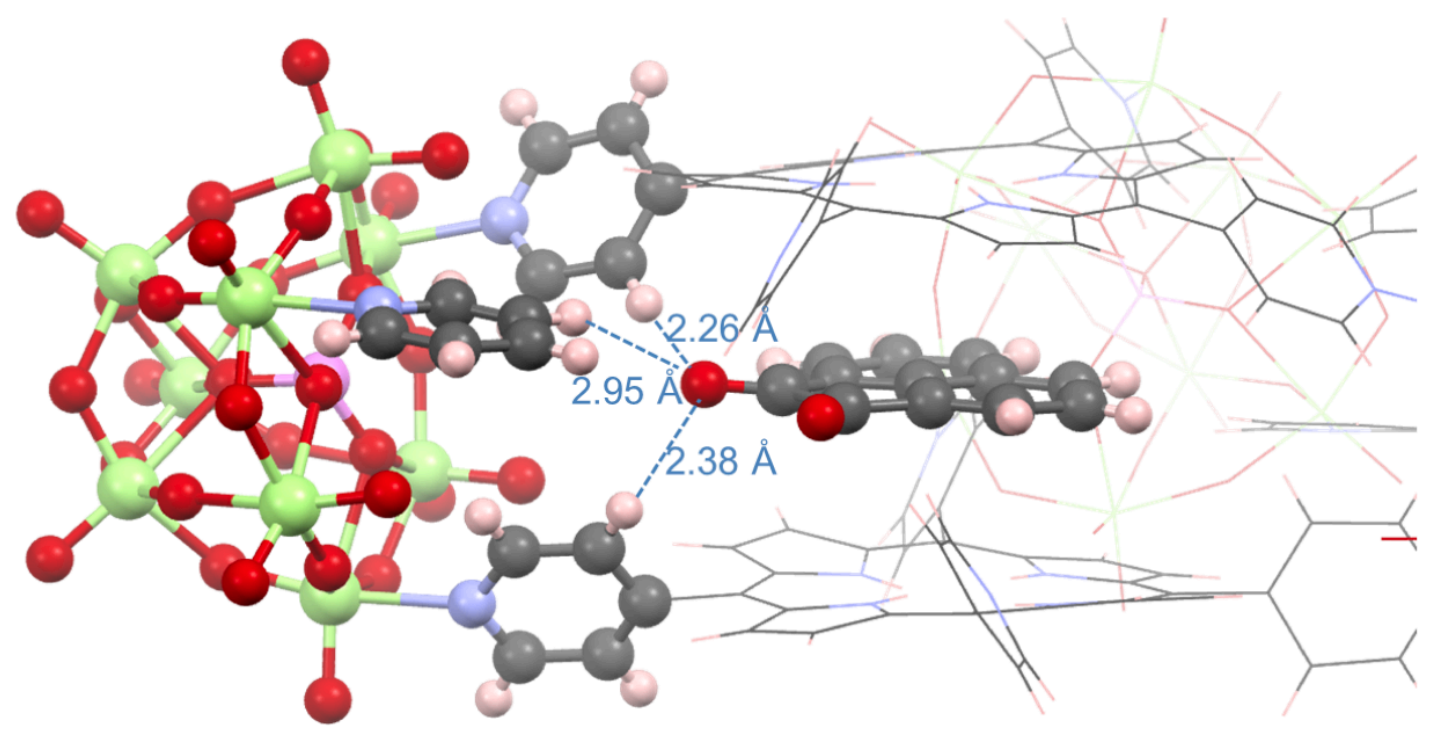

Figure S20. Representation of hydrogen bonds (blue dashed line) between the $\mathrm{C}-\mathrm{H}$ bonds of pyridyl groups of tpyp and py ligands, with the carbonyl oxygen atoms of ANQ in TPP-III•ANQ. The two carbonyl groups of ANQ point toward the py ligands and are possibly stabilized by the multiple hydrogen bonds between the $\mathrm{C}-\mathrm{H}$ of both the typy ligands and the py ligands with the carbonyl oxygen atoms of ANQ (C-H $\cdots \mathrm{O}$ distance; 2.2 to $3.3 \AA$ ). P purple, Mo green; spheres: $\mathrm{C}$ black, H pink, N light blue, $\mathrm{O}$ red. A part of the structure is shown by wire-frame for clarity.

\section{References}

S1. CrystalClear 1.3.6, Rigaku and Rigaku/MSC, The Woodlands, TX.

S2. Pflugrath, J. W. Acta Crystallogr. Sect. D 55, 1718 (1999).

S3. Otwinowski, W. Minor, Processing of X-ray Diffraction Data Collected in Oscillation Mode in Methods in Enzymology, (Eds.: C. W. Carter, Jr., R. M. Sweet), Macromolecular Crystallography, Part A, Academic press, New York, 276, 307 (1997).

S4. CrystalStructure 3.8, Rigaku and Rigaku/MSC, The Woodlands, TX.

S5. Farrugia, L. J. J. Appl. Crystallogr, 32, 837 (1999).

S6. Sheldrick, G. M. SHELX97, Programs for Crystal Structure Analysis, Release 97-92, University of Gçttingen, Gçttingen, Germany, 1997.

S7. Sheldrick, G. M. SHELX-2014, Programs for Crystal Structure Analysis, University of Gçttingen,

Gçttingen, Germany, 2014.

S8. van der Sluis, P. A. \& Spek, L. Acta Crystallogr. Sect. A 46, 194 (1990).

S9. Brown, I. D. \& Altermatt, D. Acta Crystallogr. Sect. B 41, 244 (1985).

S10. Brese, N. E. \& O’Keeffe, M. Acta Crystallogr. Sect. B 47, 192 (1991). 